\title{
Probing the Weizsäcker-Williams gluon Wigner distribution in $p p$ collisions
}

\author{
Renaud Boussarie, ${ }^{1}$ Yoshitaka Hatta, ${ }^{2}$ Bo-Wen Xiao, ${ }^{3,4}$ and Feng Yuan ${ }^{5}$ \\ ${ }^{1}$ Institute of Nuclear Physics, Polish Academy of Sciences, \\ Radzikowskiego 152, PL-31-342 Kraków, Poland \\ ${ }^{2}$ Yukawa Institute for Theoretical Physics, Kyoto University, Kyoto 606-8502, Japan \\ ${ }^{3}$ Key Laboratory of Quark and Lepton Physics (MOE) and Institute of Particle Physics, \\ Central China Normal University, Wuhan 430079, China \\ ${ }^{4}$ Centre de Physique Théorique, École Polytechnique, CNRS, Université Paris-Saclay, \\ Route de Saclay, 91128 Palaiseau, France \\ ${ }^{5}$ Nuclear Science Division, Lawrence Berkeley National Laboratory, Berkeley, California 94720, USA
}

(Received 10 August 2018; published 17 October 2018)

\begin{abstract}
We show that the diffractive forward production of two quarkonia, especially the $\chi_{c J}$ and $\eta_{c}$ states, in proton-proton or proton-ion collisions can access the Weizsäcker-Williams gluon Wigner distribution of the proton. We use the hybrid factorization approach where the collinear, double gluon PDF is applied for one of the protons and the $k_{T}$-dependent (Wigner or GTMD) distribution for the other. The production of quarkonia is treated in the NRQCD framework. A particularly concise formula is obtained for double $\chi_{J=1}$ production.
\end{abstract}

DOI: 10.1103/PhysRevD.98.074015

\section{INTRODUCTION}

In hadron physics research, one of the ultimate goals is to depict the colorful and kaleidoscopic multidimensional landscape of the internal structure of hadrons including nucleons and nucleus. In particular, in addition to the longitudinal momentum distribution of partons inside hadrons as given by the Feynman parton distributions, we also intend to learn about the transverse spatial [generalized parton distributions (GPD)] and transverse momentum [transverse momentum dependent (TMD)] distributions. The so-called quantum phase space Wigner distributions [1-3] encode all the above important information and are viewed as the mother distributions of all. In practice, since it is rather difficult to directly measure the spatial transverse coordinates in high energy scatterings, we also normally define the generalized transverse momentum distribution (GTMD) [4-6] as the Fourier transform of the corresponding Wigner distribution for quarks and gluons.

Interestingly, as pointed out in Refs. [7-11], quark and gluon TMDs are not unique due to different possible structures of gauge links representing the initial and final state interactions. As far as the gluon TMDs are concerned, there are at least two nontrivial gluon distributions whose difference becomes crucial especially in the small- $x$ region. One is known as the dipole gluon distribution which

Published by the American Physical Society under the terms of the Creative Commons Attribution 4.0 International license. Further distribution of this work must maintain attribution to the author(s) and the published article's title, journal citation, and DOI. Funded by SCOAP ${ }^{3}$. contains the dipole-shaped configuration of gauge links, while the other is called the Weizsäcker-Williams (WW) gluon distribution which features either a future-pointing or past-pointing gauge link, but not both. Naturally, one expects that this kind of complication should also persist in the case of Wigner distributions and GTMDs.

Generally speaking, up to now, the HERA experiment has provided us with the most precise knowledge of Feynman parton distributions for a large range of $x$ region, through the inclusive deep inelastic scattering (DIS) process. Furthermore, less inclusive processes, such as the semi-inclusive DIS (SIDIS) and the deeply virtual Compton scattering (DVCS) can help us gain insights on the TMDs and GPDs, respectively. The important question here is whether one can probe the Wigner distributions or GTMDs experimentally. It is rather challenging to achieve such a goal because, obviously, one has to consider more exclusive/complicated processes than SIDIS and DVCS.

Recently, we have found that the goal of measuring the gluon Wigner distributions can be achieved at the future electron-hadron colliders [12], such as the planned electron-ion colliders (EIC) and the large hadron electron collider (LHeC) [13-15]. Specifically, the dipole gluon Wigner or GTMD can be probed in diffractive dijet process in the small- $x$ region at EIC [16]. Subsequently, there have been a lot of progress on this topic in the last two years [17-28]. Especially, one can access and study the extremely evasive gluon orbital angular momentum with the help of the spin-dependent gluon GTMD as discussed in Refs. [20,21,23,27,29-31]. 
On the other hand, it has been realized already in Ref. [12] that the Weizsäcker-Williams Wigner (WWW) distribution is more difficult to access. One possibility, as suggested in [12], was to look at a process involving two incoming photons and four outgoing jets at EIC. However, the transition rate would be too small to make it a realistic measurement. The objective of this paper is to show that it is possible to rather directly probe the WWW distribution and WW type GTMD for small- $x$ gluons in the production of two heavy scalar quarkonia such as $\chi_{c}$ and $\eta_{c}$ in the forward rapidity region in diffractive $p p$ and $p A$ collisions $p p, p A \rightarrow \chi_{c} \chi_{c} p X$.

The rest of the paper is organized as follows. We first introduce the WW gluon GTMD and briefly describe the process we will consider in this paper, i.e., double quarkonia production in the hard diffractive processes in $p p$ and $p A$ collisions. In Sec. III, we derived the amplitude for the double quarkonia production through double gluon scattering on the nucleon/nucleus targets. These amplitudes will be converted into $p p / p A$ collisions cross sections in Sec. IV by applying the double parton scattering framework for the incoming two gluons from the projectile. In Sec. III and IV, we derive the results for both $\eta$ and $\chi$. In particular, we will present the explicit differential cross sections on different combinations of two quarkonia states of $\eta, \chi_{0,1,2}$. Finally, we summarize our paper in Sec. VI.

\section{WEIZSÄCKER-WILLIAMS GLUON GTMD}

We start by writing down the Weizsäcker-Williams (WW) gluon GTMD. For the "target" proton fast-moving in the negative $z$-direction we define

$$
\begin{aligned}
x \mathcal{G}^{i j}(\boldsymbol{K}, \boldsymbol{\Delta}) \equiv & 2 \int \frac{d^{3} z}{P^{-}(2 \pi)^{3}} e^{i x P^{-} z^{+}-i \boldsymbol{K} \cdot z} \\
& \times\left\langle P-\frac{\Delta}{2}\right| \operatorname{Tr}\left[U_{ \pm}^{\dagger} F^{-i}(z / 2) U_{ \pm}\right. \\
& \left.\times F^{-j}(-z / 2)\right]\left|P+\frac{\Delta}{2}\right\rangle,
\end{aligned}
$$

where $U_{ \pm}$is the staple-shaped fundamental Wilson line connecting the points $z / 2$ and $-z / 2$ via light-like Wilson lines encircling $x^{+}= \pm \infty$. Boldface letters denote twodimensional vectors. We have kept the transverse indices $i$, $j=1,2$ open because this is what we shall need in later calculations. In the small- $x$ region, we may approximate $e^{i x P^{-} z^{+}} \approx 1$ and find (cf., [11])

$$
\begin{aligned}
x \mathcal{G}^{i j}(\boldsymbol{K}, \boldsymbol{\Delta}) \approx & -\frac{2}{\alpha_{s}} \int \frac{d^{2} \boldsymbol{b}_{1} d^{2} \boldsymbol{b}_{2}}{(2 \pi)^{4}} e^{-i \boldsymbol{\Delta} \cdot \frac{\boldsymbol{b}_{1}+\boldsymbol{b}_{2}}{2}-i \boldsymbol{K} \cdot\left(\boldsymbol{b}_{1}-\boldsymbol{b}_{2}\right)} \\
& \times \frac{\left\langle P-\frac{\Delta}{2}\left|\operatorname{Tr}\left[\left(\partial^{i} U_{\boldsymbol{b}_{1}}^{\dagger}\right) U_{\boldsymbol{b}_{1}}\left(\partial^{j} U_{\boldsymbol{b}_{2}}^{\dagger}\right) U_{\boldsymbol{b}_{2}}\right]\right| P+\frac{\Delta}{2}\right\rangle}{\langle P \mid P\rangle},
\end{aligned}
$$

where $U_{b}$ is the lightlike Wilson line from $x^{+}=-\infty$ to $x^{+}=+\infty$ at fixed transverse position $\boldsymbol{b}$. It is easy to check from (2) the relations $\mathcal{G}^{i j}(\boldsymbol{K}, \boldsymbol{\Delta})=\mathcal{G}^{j i}(-\boldsymbol{K}, \boldsymbol{\Delta})=$ $\left(\mathcal{G}^{i j}(-\boldsymbol{K},-\boldsymbol{\Delta})\right)^{*}$. The general parametrization is thus ${ }^{1}$

$$
\begin{aligned}
\mathcal{G}^{i j}(\boldsymbol{K}, \boldsymbol{\Delta}) \equiv & \delta^{i j} \mathcal{G}_{1}+\left(\frac{\boldsymbol{K}^{i} \boldsymbol{K}^{j}}{\boldsymbol{K}^{2}}-\frac{\delta^{i j}}{2}\right) \frac{\boldsymbol{K}^{2}}{M^{2}} \mathcal{G}_{2} \\
& +\left(\frac{\boldsymbol{\Delta}^{i} \boldsymbol{\Delta}^{j}}{\boldsymbol{\Delta}^{2}}-\frac{\delta^{i j}}{2}\right) \frac{\boldsymbol{\Delta}^{2}}{M^{2}} \mathcal{G}_{3}+\left(\frac{\boldsymbol{K}^{i} \boldsymbol{\Delta}^{j}-\boldsymbol{\Delta}^{i} \boldsymbol{K}^{j}}{M^{2}}\right) \mathcal{G}_{4},
\end{aligned}
$$

where $M$ is the nucleon mass. $\mathcal{G}_{1,2,3,4}$ are all real and depend on $\boldsymbol{K}^{2}, \boldsymbol{\Delta}^{2}$ and $(\boldsymbol{K} \cdot \boldsymbol{\Delta})^{2}$. In the forward limit $\boldsymbol{\Delta}=0, \mathcal{G}_{1}$ and $\mathcal{G}_{2}$ reduce to the unpolarized and linearly polarized WW gluon TMDs, respectively. The $\boldsymbol{K}$-integral of $\mathcal{G}_{3}$ is proportional to the gluon transversity GPD which in turn is related to the so-called elliptic gluon Wigner distribution [24]. From what we know about the latter distribution $[12,18,22]$, we presume that $\mathcal{G}_{3}$ is numerically small, on the order of a few percent effect compared to $\mathcal{G}_{1,2}$.

Various experimental processes have been identified to probe the WW gluon TMD [11]. On the other hand, the measurement of the GTMDs is more challenging, and most of the proposals so far concern the dipole gluon GTMD whose treatment is somewhat simpler because of its relation to the dipole S-matrix [12]. In this paper, we show that double quarkonium production in $p p$ collisions $p p \rightarrow M_{1} M_{2} p X$ is a very sensitive observable for the WW gluon GTMD. This is a natural extension of the previous observation [33] (see, also, [34]) that the WW gluon TMD can be probed in single quarkonium production $p p \rightarrow M X$. The hard subprocess and the full process are respectively depicted in Fig. 1 and in Fig. 2. Two gluons are emitted from the projectile proton and scatter off the shockwave field created by the target proton. In the final state, we measure two quarkonia with momentum $K_{1}$ and $K_{2}$ in the forward region as well as the elastically scattered target with momentum transfer $\boldsymbol{\Delta}=\boldsymbol{K}_{1}+\boldsymbol{K}_{2}$ in the backward region. To lowest order, the shockwave consists of two gluons in the $t$-channel, and we can view this diagram as the square of the $g g^{*} \rightarrow M$ amplitude. This means that the produced quarkonia must be $C$-even states, such as $\eta_{c}$ and $\chi_{c J}$ (and their bottomonium counterparts). We study this process in the "hybrid factorization" approach (collinear gluons from one proton and noncollinear gluons from the other proton) and show that the cross section can be written as a convolution of the square of the WW gluon GTMD of the target and the double gluon PDF of the projectile.

It should be mentioned that a very similar idea came out recently in Ref. [31] where the authors proposed to measure the gluon GTMD (including its spin dependence) in doubly-diffractive double $\eta_{c}$ production $p p \rightarrow p p \eta_{c} \eta_{c}$ for moderate values of $x$. Their argument is limited to the two-gluon exchange level where the difference between

${ }^{1}$ Naively, there is another term proportional to the tensor structure $\boldsymbol{K}^{i} \boldsymbol{\Delta}^{j}+\boldsymbol{K}^{j} \boldsymbol{\Delta}^{i}-\boldsymbol{K} \cdot \boldsymbol{\Delta} \delta^{i j}$. However, as pointed out in [32], this term is not independent and can be absorbed in $\mathcal{G}_{2,3}$. See, also, [5]. 


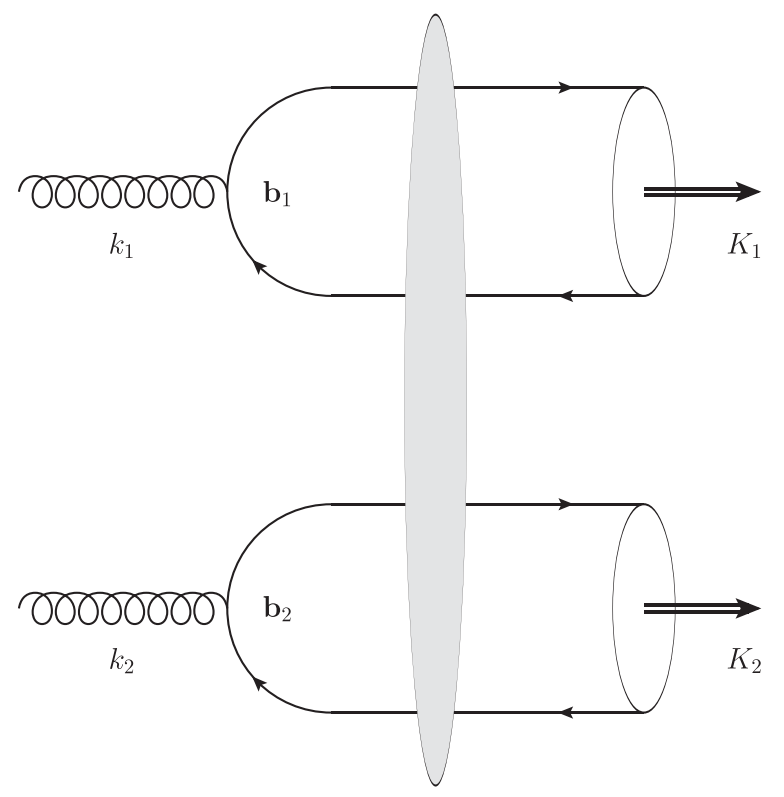

FIG. 1. Production of 2 quarkonia from 2 gluons.

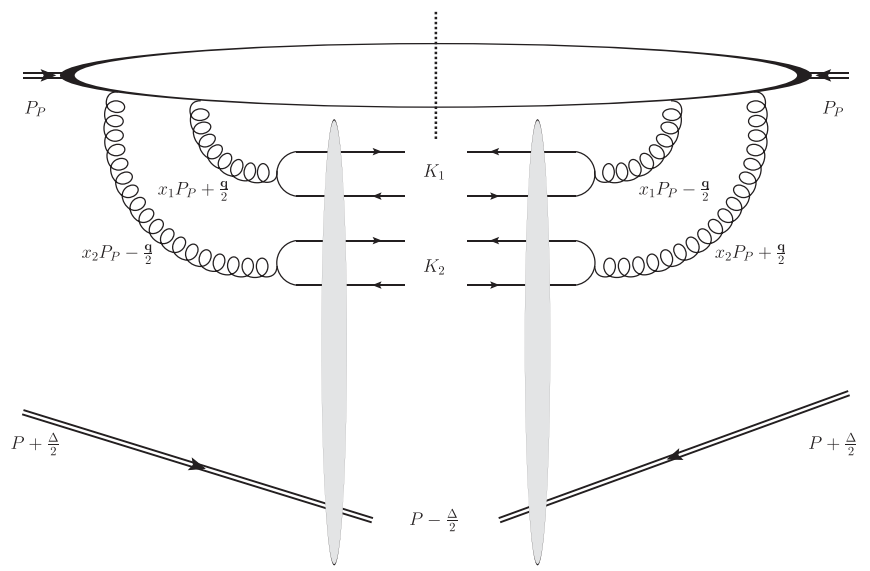

FIG. 2. Production of a quarkonium pair in hybrid factorization with double scattering on the projectile side.

WW and dipole distributions becomes immaterial. Our process is single-diffractive and we do not consider spin effects. Instead, we focus on the forward, small- $x$ region and carefully examine the structure of the relevant Wilson lines. Our result suggests that the GTMD discussed in [31] likely becomes the WW GTMD once higher order rescattering effects are taken into account.

\section{QUARKONIUM PRODUCTION AMPLITUDE}

\section{A. Single quarkonium production}

As a warm-up, we first compute the amplitude for the single quarkonium production in the hybrid factorization approach combined with the nonrelativistic QCD (NRQCD) framework. Related calculations can be found in the literature, e.g., [33,34]. The right-moving projectile

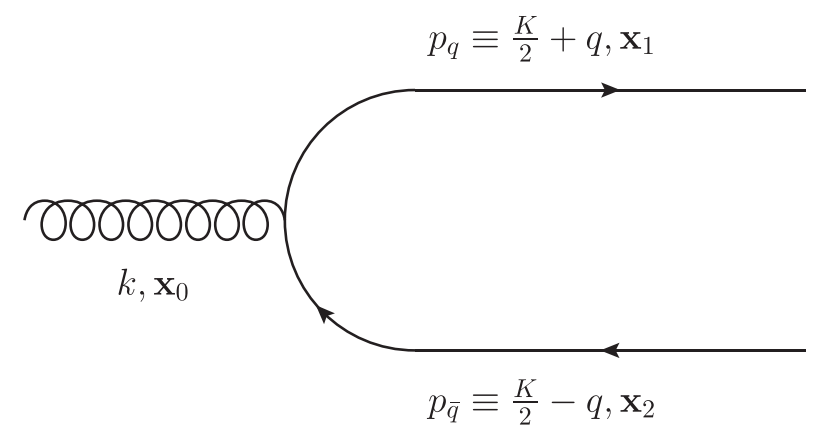

FIG. 3. Open charm production.

proton is treated as a dilute object, i.e., partons are extracted from the projectile using regular parton distribution functions, whereas the left-moving target is treated as a shockwave. The subamplitude for the open production of a massive $q \bar{q}$ pair from a gluon (see Fig. 3) can be straightforwardly written as, in $D=d+2$ dimensions, ${ }^{2}$

$$
\left(\mathcal{A}_{\sigma}\right)^{c}=-i g \int d^{D} z_{0} \bar{u}\left(p_{q}, z_{0}\right) \gamma^{\mu} t^{b} \varepsilon_{\mu}^{c b}\left(k, z_{0}\right) v\left(p_{\bar{q}}, z_{0}\right),
$$

where, with similar methods as in $[35,36]$ one can derive the effective quark, antiquark, and gluon lines as obtained for example in $[17,37]$ :

$$
\begin{aligned}
\bar{u}\left(p_{q}, z_{0}\right)= & \frac{1}{2}\left(\frac{p_{q}^{+}}{2 \pi}\right)^{\frac{d}{2}} \int d^{d} \boldsymbol{x}_{1} e^{i p_{q}^{+}\left(z_{0}^{-}-\frac{\left(x_{1}-z_{0}\right)^{2}}{2 z_{0}^{+}}+i 0\right)-i p_{q} \cdot x_{1}+\frac{z_{0}^{+}}{2 p_{q}^{+}}\left(m^{2}+i 0\right)} \\
& \times\left(\frac{i}{z_{0}^{+}}\right)^{\frac{d}{2}} \bar{u}_{p_{q}} \gamma^{+}\left[U_{x_{1}} \theta\left(-z_{0}^{+}\right)+\theta\left(z_{0}^{+}\right)\right] \\
& \times\left(\gamma^{-}-\frac{\not x_{1 \perp}-\not_{0 \perp}}{z_{0}^{+}}+\frac{m}{p_{q}^{+}}\right)
\end{aligned}
$$

$$
\begin{aligned}
v\left(p_{\bar{q}}, z_{0}\right)= & \frac{1}{2}\left(\frac{p_{\bar{q}}^{+}}{2 \pi}\right)^{\frac{d}{2}} \int d^{d} \boldsymbol{x}_{2} e^{i p_{\bar{q}}^{+}\left(z_{0}^{-}-\frac{\left(x_{2}-z_{0}\right)^{2}}{2 z_{0}^{+}}+i 0\right)-i p_{\bar{q}} \cdot x_{2}+i \frac{z_{0}^{+}}{2 p_{\bar{q}}^{+}}\left(m^{2}+i 0\right)} \\
& \times\left(\frac{i}{z_{0}^{+}}\right)^{\frac{d}{2}}\left(\gamma^{-}-\frac{\not x_{2 \perp}-\not t_{0 \perp}}{z_{0}^{+}}-\frac{m}{p_{\bar{q}}^{+}}\right) \\
& \times\left[U_{\boldsymbol{x}_{2}}^{\dagger} \theta\left(-z_{0}^{+}\right)+\theta\left(z_{0}^{+}\right)\right] \gamma^{+} v_{p_{\bar{q}}}
\end{aligned}
$$

\footnotetext{
${ }^{2}$ Our notation is as follows: We define two lightlike vectors $n_{1}, n_{2}$ such that the projectile flies along $n_{1}$ and the target flies along $n_{2}$, and such that $n_{1} \cdot n_{2}=1$. Lightcone coordinates are then defined as

$$
k^{\mu} \equiv k^{+} n_{1}^{\mu}+k^{-} n_{2}^{\mu}+k_{\perp}^{\mu},
$$$$
k \cdot l \equiv k^{+} l^{-}+k^{-} l^{+}+k_{\perp} \cdot l_{\perp} \equiv k^{+} l^{-}+k^{-} l^{+}-(\boldsymbol{k} \cdot \boldsymbol{l}) .
$$

The metric tensor will be decomposed into its longitudinal and transverse parts as

$$
g^{\mu \nu} \equiv n_{1}^{\mu} n_{2}^{\nu}+n_{1}^{\nu} n_{2}^{\mu}+g_{\perp}^{\mu \nu} .
$$




$$
\begin{aligned}
\varepsilon_{\mu}^{b a}\left(k, z_{0}\right)= & \left(\frac{k^{+}}{2 \pi}\right)^{\frac{d}{2}} \int d^{d} \boldsymbol{x}_{0} e^{-i k^{+}\left(z_{0}^{-}-\frac{\left(x_{0}-z_{0}\right)^{2}}{2 z_{0}^{+}}-i 0\right)+i \boldsymbol{k} \cdot \boldsymbol{x}_{0}} \\
& \times\left(\frac{-i}{z_{0}^{+}}\right)^{\frac{d}{2}}\left(g_{\perp \mu \sigma}+\frac{x_{0 \perp \sigma}-z_{0 \perp \sigma}}{z_{0}^{+}} n_{2 \mu}\right) \\
& \times\left[U_{\boldsymbol{x}_{0}}^{b a} \theta\left(z_{0}^{+}\right)+\delta^{a b} \theta\left(-z_{0}^{+}\right)\right] \varepsilon_{k \perp}^{\sigma}
\end{aligned}
$$

In the above, $m$ is the heavy quark mass and we have kept the transverse momentum of the incoming gluon $\boldsymbol{k}$. $U$ is the Wilson line which arises after the eikonal interaction with the target field $A^{-}$

$$
U_{\boldsymbol{x}} \equiv \mathcal{P} e^{i g \int_{-\infty}^{\infty} d x^{+} A^{-}(x)}
$$

We will use the conventions that lines with color indices as superscripts $U_{\boldsymbol{x}}^{a b}$ are in the adjoint representation while lines without color indices are in the fundamental representation.

Integrating over the interaction point $z_{0}$, we get

$$
\left(\mathcal{A}_{\sigma}\right)^{c}=\varepsilon_{k \perp}^{\sigma} \bar{u}_{p_{q}} \mathcal{M}_{\sigma}^{c} v_{p_{\bar{q}}}
$$

where

$$
\begin{aligned}
\mathcal{M}_{\sigma}^{c}= & -\frac{i g}{2}(2 \pi) \delta\left(p_{q}^{+}+p_{\bar{q}}^{+}-k^{+}\right)\left(\frac{p_{q}^{+} p_{\bar{q}}^{+} k^{+}}{2 \pi}\right)^{\frac{d}{2}} \int d^{d} \boldsymbol{x}_{1} d^{d} \boldsymbol{x}_{2} d^{d} \boldsymbol{x}_{0} e^{-i p_{q} \cdot \boldsymbol{x}_{1}-i p_{\bar{q}} \cdot \boldsymbol{x}_{2}+i \boldsymbol{k} \cdot \boldsymbol{x}_{0}} \delta\left(p_{q}^{+} \boldsymbol{x}_{1}+p_{\bar{q}}^{+} \boldsymbol{x}_{2}-k^{+} \boldsymbol{x}_{0}\right) \\
& \times\left[\frac{1}{z_{0}^{+}}\left(\gamma_{\perp \sigma} x_{12 \perp}-2 \frac{p_{\bar{q}}^{+}}{k^{+}} x_{12 \perp \sigma}\right)-m \frac{k^{+}}{p_{q}^{+} p_{\bar{q}}^{+}} \gamma_{\perp \sigma}\right] \gamma^{+}\left[(-i)^{\frac{d}{2}} \int_{-\infty}^{0} d z_{0}^{+}\left(-z_{0}^{+}\right)^{-\frac{d}{2}}\left(U_{\boldsymbol{x}_{1}} t^{c} U_{\boldsymbol{x}_{2}}^{\dagger}\right) e^{i \frac{k^{+}\left(m^{2}-i 0\right)}{2 p_{q}^{+} p_{\bar{q}}^{+}} z_{0}^{+}-i \frac{p_{q}^{+} p_{\bar{q}}^{+} x_{121+i 0}^{2}}{2 k^{+}} z_{0}^{+}}\right. \\
& \left.+i^{\frac{d}{2}} \int_{0}^{+\infty} d z_{0}^{+}\left(z_{0}^{+}\right)^{-\frac{d}{2}}\left(t^{d} U_{\boldsymbol{x}_{0}}^{c d}\right) e^{i \frac{k^{+}\left(m^{2}+i 0\right)}{2 p_{q}^{+} p_{\bar{q}}^{+}} z_{0}^{+}-i \frac{p_{q}^{+} p_{\bar{q}}^{+} x_{121-i 0}^{2}}{2 k^{+}} z_{0}^{+}}\right]
\end{aligned}
$$

Using the integrals, for $Q, Z>0$ :

$$
\begin{aligned}
& \int_{-\infty}^{0} d z^{+}\left(-z^{+}\right)^{-n} e^{i(Q-i 0) z^{+}-i \frac{Z+i 0}{z^{+}}}=2(-i)^{1-n}\left(\sqrt{\frac{Z}{Q}}\right)^{1-n} K_{n-1}(2 \sqrt{Q Z}) \\
& \int_{0}^{+\infty} d z^{+}\left(z^{+}\right)^{-n} e^{i(Q+i 0) z^{+}-i \frac{Z-i 0}{z^{+}}}=2 i^{1-n}\left(\sqrt{\frac{Z}{Q}}\right)^{1-n} K_{n-1}(2 \sqrt{Q Z}),
\end{aligned}
$$

we get

$$
\begin{aligned}
\mathcal{M}_{\sigma}^{c}= & i g \delta\left(p_{q}^{+}+p_{\bar{q}}^{+}-k^{+}\right) \int d^{d} \boldsymbol{x}_{1} d^{d} \boldsymbol{x}_{2} d^{d} \boldsymbol{x}_{0} e^{-i p_{q} \cdot \boldsymbol{x}_{1}-i p_{\bar{q}} \cdot \boldsymbol{x}_{2}+i \boldsymbol{k} \cdot \boldsymbol{x}_{0}} \\
& \times(2 \pi) \delta\left(p_{q}^{+} \boldsymbol{x}_{1}+p_{\bar{q}}^{+} \boldsymbol{x}_{2}-k^{+} \boldsymbol{x}_{0}\right)\left(\frac{m\left(k^{+}\right)^{2}}{2 \pi\left|\vec{x}_{12}\right|}\right)^{\frac{d}{2}}\left(U_{\boldsymbol{x}_{1}} t^{c} U_{\boldsymbol{x}_{2}}^{\dagger}-t^{d} U_{\boldsymbol{x}_{0}}^{c d}\right) \\
& \times\left[K_{\frac{d}{2}}\left(m\left|\boldsymbol{x}_{12}\right|\right)\left(\gamma_{\perp \sigma} x_{12 \perp}-2 \frac{p_{\bar{q}}^{+}}{k^{+}} x_{12 \perp \sigma}\right)-i\left|\boldsymbol{x}_{12}\right| K_{\frac{d}{2}-1}\left(m\left|\boldsymbol{x}_{12}\right|\right) \gamma_{\perp \sigma}\right] \gamma^{+} .
\end{aligned}
$$

In four dimensions and after a few simple changes of variables, this can finally be rewritten as the known result:

$$
\begin{aligned}
\mathcal{M}_{\sigma}^{c}= & i m g \delta\left(K^{+}-k^{+}\right) \int d^{2} \boldsymbol{b} d^{2} \boldsymbol{r} e^{-i(\boldsymbol{K}-\boldsymbol{k}) \cdot \boldsymbol{b}+i q^{+}\left(\frac{\boldsymbol{k}}{K^{+}}-\frac{q}{q^{+}}\right) \cdot \boldsymbol{r}}\left(U_{\boldsymbol{b}+\frac{r}{2}} c^{c} U_{\boldsymbol{b}-\frac{r}{2}}^{\dagger}-t^{d} U_{\boldsymbol{b}}^{c d}\right) \\
& \times\left[\frac{\gamma_{\perp \sigma} \gamma_{\perp}-\left(1-2 \frac{q^{+}}{K^{+}}\right) r_{\perp \sigma}}{|\boldsymbol{r}|} K_{1}(m|\boldsymbol{r}|)-i K_{0}(m|\boldsymbol{r}|) \gamma_{\perp \sigma}\right] \gamma^{+}
\end{aligned}
$$

Our next step is to convolute this amplitude with the transition probability to a quarkonium in the NRQCD approach where one expands the charmonium wave function as a series in powers of the relative velocity of its constituents. As we have noted already, we shall be interested in $C$-even quarkonia, $\eta$ and $\left(\chi_{J}\right)_{J=0,1,2}$. The corresponding projectors for the pseudoscalar $n^{2 s+1} L_{0}$ and vector $n^{2 s+1} L_{1}$ states are 


$$
\begin{aligned}
& \Pi_{0} \equiv \frac{1}{\sqrt{8 m^{3}}}\left(\frac{K}{2}-\not-m\right) \gamma_{5}\left(\frac{K}{2}+q+m\right), \\
& \Pi_{1}^{\rho} \equiv \frac{1}{\sqrt{8 m^{3}}}\left(\frac{K}{2}-q-m\right) \gamma^{\rho}\left(\frac{K}{2}+q+m\right) .
\end{aligned}
$$

Then the perturbative part of the ${ }^{1} S_{0}$ wave and ${ }^{3} P_{J}$ wave transitions read respectively:

$$
\begin{aligned}
\mathcal{A}^{\sigma, c}\left({ }^{1} S_{0}\right) & \equiv\left[\operatorname{Tr}\left(\Pi_{0}\left(\mathcal{M}^{\sigma}\right)^{c}\right)\right]_{q=0} \\
& =\sqrt{2 m} g \delta\left(K^{+}-k^{+}\right) K^{+} \int d^{2} \boldsymbol{r} d^{2} \boldsymbol{b} e^{-i(\boldsymbol{K}-\boldsymbol{k}) \cdot \boldsymbol{b}}\left(U_{\boldsymbol{b}+\frac{r}{2}} t^{c} U_{\boldsymbol{b}-\frac{r}{2}}^{\dagger}-U_{\boldsymbol{b}} t^{c} U_{\boldsymbol{b}}^{\dagger}\right) \epsilon^{\sigma_{\perp} \mu_{\perp}+-} \frac{r_{\perp \mu}}{|\boldsymbol{r}|} K_{1}(m|\boldsymbol{r}|),
\end{aligned}
$$

and

$$
\begin{aligned}
\mathcal{A}^{\sigma, c}\left({ }^{3} P_{J}\right) \equiv & \varepsilon_{(J)}^{\rho \mu}\left[\frac{d}{d q^{\rho}} \operatorname{Tr}\left(\Pi_{1 \mu}\left(\mathcal{M}^{\sigma}\right)^{c}\right)\right]_{q=0} \\
= & i g \sqrt{2 m} \varepsilon_{(J) \rho \mu} \delta\left(K^{+}-k^{+}\right) \int d^{2} \boldsymbol{r} d^{2} \boldsymbol{b} e^{-i(\boldsymbol{K}-\boldsymbol{k}) \cdot \boldsymbol{b}}\left\{r_{\perp \alpha} K_{0}(m|\boldsymbol{r}|)\left(K^{+} g_{\perp}^{\sigma \mu}-K_{\perp}^{\sigma} n_{2}^{\mu}\right)\right. \\
& \times\left[\left(g_{\perp}^{\alpha \rho}-\frac{k_{\perp}^{\alpha}}{K^{+}} n_{2}^{\rho}\right)\left(U_{\boldsymbol{b}+\frac{r}{2}} t^{c} U_{\boldsymbol{b}-\frac{r}{2}}^{\dagger}\right)-\left(g_{\perp}^{\alpha \rho}-\frac{K_{\perp}^{\alpha}}{K^{+}} n_{2}^{\rho}\right)\left(U_{\boldsymbol{b}} t^{c} U_{\boldsymbol{b}}^{\dagger}\right)\right]+\frac{r_{\perp \alpha} K_{1}(m|\boldsymbol{r}|)}{m|\boldsymbol{r}|}\left(U_{\boldsymbol{b}+\frac{r}{2}} t^{c} U_{\boldsymbol{b}-\frac{r}{2}}^{\dagger}-U_{\boldsymbol{b}} t^{c} U_{\boldsymbol{b}}^{\dagger}\right) \\
& \left.\times\left[K^{+}\left(g_{\perp}^{\rho \sigma} g_{\perp}^{\alpha \mu}-g_{\perp}^{\alpha \rho} g_{\perp}^{\sigma \mu}\right)+\left(K^{\mu} g_{\perp}^{\alpha \sigma}+K_{\perp}^{\alpha} g_{\perp}^{\sigma \mu}-K_{\perp}^{\sigma} g_{\perp}^{\alpha \mu}\right) n_{2}^{\rho}+\left(K_{\perp}^{\sigma} g_{\perp}^{\alpha \rho}-K_{\perp}^{\alpha} g_{\perp}^{\rho \sigma}-g_{\perp}^{\alpha \sigma} \frac{m^{2}}{K^{+}} n_{2}^{\rho}\right) n_{2}^{\mu}\right]\right\} .
\end{aligned}
$$

Next we use the fact that the modified Bessel functions are peaked around 0 to expand the integrand as

$$
\int d^{2} \boldsymbol{r} K_{n}(m \mid \boldsymbol{r}) F(\boldsymbol{r}) \approx \int d^{2} \boldsymbol{r}\left[K_{n}(m|\boldsymbol{r}|) F(\mathbf{0})+K_{n}(m|\boldsymbol{r}|) \partial_{\perp}^{\mu} \partial_{\mu} F(\mathbf{0})\right]
$$

and perform the $d^{2} \boldsymbol{r}$ integrals

$$
\int d^{2} \boldsymbol{r} K_{0}(m|\boldsymbol{r}|) r_{\perp \alpha} r_{\perp}^{\nu}=-\frac{4 \pi}{m^{4}} g_{\perp \alpha}^{\nu} \quad \int d^{2} \boldsymbol{r} \frac{K_{1}(m|\boldsymbol{r}|)}{m|\boldsymbol{r}|} r_{\perp \alpha} r_{\perp}^{\nu}=-\frac{2 \pi}{m^{4}} g_{\perp \alpha}^{\nu} .
$$

Finally, we notice that the leading NRQCD contribution for $\eta$ mesons and for $\chi_{J}$ mesons are color singlet contributions for ${ }^{1} S_{0}$ and for ${ }^{3} P_{J}$ waves, respectively. Introducing the NRQCD long distance matrix elements (LDME's) and the color singlet projector

$$
\frac{\delta^{i j}}{N_{c}}\left\langle\mathcal{O}_{\eta}\left({ }^{1} S_{0}^{1}\right)\right\rangle^{\frac{1}{2}}, \quad \frac{\delta^{i j}}{N_{c}}\left\langle\mathcal{O}_{\chi_{J}}\left({ }^{3} P_{J}^{1}\right)\right\rangle^{\frac{1}{2}},
$$

( $i j$ are color indices and the second superscript " 1 " denotes color singlet) we arrive at the gluon-to-meson transition amplitudes:

$$
\mathcal{A}^{\sigma, c}(\eta)=\frac{2 g \pi}{m^{3}} \sqrt{2 m} \delta\left(K^{+}-k^{+}\right) \int d^{2} \boldsymbol{b} e^{-i(\boldsymbol{K}-\boldsymbol{k}) \cdot \boldsymbol{b}} \frac{1}{N_{c}} \operatorname{Tr}\left[\left(\partial_{\nu} U_{\boldsymbol{b}}^{\dagger}\right) U_{\boldsymbol{b}} t^{c}\right] K^{+} \epsilon^{\sigma_{\perp} \nu_{\perp}+-}\left\langle\mathcal{O}_{\eta}\left({ }^{1} S_{0}^{1}\right)\right\rangle^{\frac{1}{2}}
$$

for $\eta$ transitions, and

$$
\mathcal{A}^{\sigma, c}\left(\chi_{J}\right)=\frac{2 g \pi}{m^{3}} \sqrt{2 m} \delta\left(K^{+}-k^{+}\right) \int d^{2} \boldsymbol{b} e^{-i(\boldsymbol{K}-\boldsymbol{k}) \cdot \boldsymbol{b}} \frac{1}{N_{c}} \operatorname{Tr}\left[\left(\partial_{\alpha} U_{\boldsymbol{b}}^{\dagger}\right) U_{\boldsymbol{b}} t^{c}\right] \frac{i}{m} \varepsilon_{(J) \rho \mu} \mathcal{P}^{\sigma \alpha \rho \mu}\left\langle\mathcal{O}_{\chi_{J}}\left({ }^{3} P_{J}^{1}\right)\right\rangle^{\frac{1}{2}},
$$

for $\chi_{J}$ transitions. We used the transversity condition $K^{\rho} \varepsilon_{J \rho \mu}=0$ to obtain (25). The tensor structure in (25) reads

$$
\mathcal{P}^{\sigma \alpha \mu \rho} \equiv K^{+}\left(g_{\perp}^{\sigma \mu} g_{\perp}^{\alpha \rho}+g_{\perp}^{\alpha \mu} g_{\perp}^{\rho \sigma}\right)-\left(K_{\perp}^{\sigma} g_{\perp}^{\alpha \rho}+K_{\perp}^{\alpha} g_{\perp}^{\rho \sigma}\right) n_{2}^{\mu}+\left[\left(K_{\perp}^{\alpha}-2 k_{\perp}^{\alpha}\right) g_{\perp}^{\sigma \mu}-K_{\perp}^{\sigma} g_{\perp}^{\alpha \mu}\right] n_{2}^{\rho}+\frac{2}{K^{+}}\left(k_{\perp}^{\alpha} K_{\perp}^{\sigma}-2 m^{2} g_{\perp}^{\alpha \sigma}\right) n_{2}^{\mu} n_{2}^{\rho}
$$

We shall use the following compact notation which summarizes the above results

$$
\mathcal{A}^{\sigma, c}\left({ }^{2 S+1} L_{J}\right) \equiv \frac{2 g \pi}{m^{3}} \sqrt{2 m} \delta\left(K^{+}-k^{+}\right)\left\langle\mathcal{O}_{M}\left({ }^{2 S+1} L_{J}^{1}\right)\right\rangle^{\frac{1}{2}} \int d^{2} \boldsymbol{b} e^{-i(\boldsymbol{K}-\boldsymbol{k}) \cdot \boldsymbol{b}} \frac{1}{N_{c}} \operatorname{Tr}\left[\left(\partial_{\alpha} U_{\boldsymbol{b}}^{\dagger}\right) U_{\boldsymbol{b}} t^{c}\right] \mathcal{P}^{\sigma \alpha}\left({ }^{2 S+1} L_{J}\right),
$$


where the tensor structures read

$$
\begin{aligned}
\mathcal{P}^{\sigma \alpha}\left({ }^{3} S_{0}\right) & \equiv K^{+} \epsilon^{\sigma_{\perp} \alpha_{\perp}+-}, \\
\mathcal{P}^{\sigma \alpha}\left({ }^{3} P_{J}\right) & \equiv \frac{i}{m} \varepsilon_{(J) \rho \mu} \mathcal{P}^{\sigma \alpha \rho \mu} .
\end{aligned}
$$

In Appendix, we compute the single-inclusive cross section of ${ }^{3} S_{0}$ and ${ }^{3} P_{J}$ states in $p p$ (or $p A$ ) collisions, in order to check the compatibility our results with the single-inclusive $J / \psi$ production computed in [34].

\section{B. Double quarkonium production}

We are now ready to write down the generic diffractive amplitude for the process $g g \rightarrow M_{1}\left({ }^{2 S_{1}+1} L_{1 J_{1}}\right) M_{2}\left({ }^{2 S_{2}+1} L_{2 J_{2}}\right)$. We basically square the $g \rightarrow M$ amplitude (27) and project onto the color singlet state. First let us assume that the final state consists of a $c \bar{c}$ quarkonium and a $b \bar{b}$ quarkonium. In this case the formula

$$
\begin{array}{r}
\operatorname{Tr}\left[U_{\boldsymbol{b}_{1}} t^{a}\left(\partial_{\alpha_{1}} U_{\boldsymbol{b}_{1}}^{\dagger}\right)\right] \operatorname{Tr}\left[U_{\boldsymbol{b}_{2}} t^{a}\left(\partial_{\alpha_{2}} U_{\boldsymbol{b}_{2}}^{\dagger}\right)\right] \\
=\frac{1}{2} \operatorname{Tr}\left[\left(\partial_{\alpha_{1}} U_{\boldsymbol{b}_{1}}^{\dagger}\right) U_{\boldsymbol{b}_{1}}\left(\partial_{\alpha_{2}} U_{\boldsymbol{b}_{2}}^{\dagger}\right) U_{\boldsymbol{b}_{2}}\right]
\end{array}
$$

immediately gives the core structure of the WW GTMD (2). The full amplitude is

$$
\begin{aligned}
& \left(\mathcal{S}^{\sigma_{1} \sigma_{2}}\right)\left(M_{1}, M_{2}\right)=\frac{4 g^{2} \pi^{2}}{\sqrt{m_{1}^{5} m_{2}^{5}}} \frac{\delta\left(K_{1}^{+}-k_{1}^{+}\right) \delta\left(K_{2}^{+}-k_{2}^{+}\right)}{N_{c}^{2}\left(N_{c}^{2}-1\right)} \\
& \times \int d^{2} \boldsymbol{b}_{1} d^{2} \boldsymbol{b}_{2} e^{-i\left(\boldsymbol{K}_{1}-\boldsymbol{k}_{1}\right) \cdot \boldsymbol{b}_{1}-i\left(\boldsymbol{K}_{2}-\boldsymbol{k}_{2}\right) \cdot \boldsymbol{b}_{2}} \\
& \times\left\langle P_{T}^{\prime}\left|\operatorname{Tr}\left[\left(\partial_{\alpha_{1}} U_{\boldsymbol{b}_{1}}^{\dagger}\right) U_{\boldsymbol{b}_{1}}\left(\partial_{\alpha_{2}} U_{\boldsymbol{b}_{2}}^{\dagger}\right) U_{\boldsymbol{b}_{2}}\right]\right| P_{T}\right\rangle \\
& \times\left\langle\mathcal{O}_{M_{1}}\left({ }^{2 S_{1}+1} L_{1 J_{1}}\right)\right\rangle^{\frac{1}{2}}\left\langle\mathcal{O}_{M_{2}}\left({ }^{2 S_{2}+1} L_{2 J_{2}}\right)\right\rangle^{\frac{1}{2}} \\
& \times \mathcal{P}^{\sigma_{1} \alpha_{1}}\left(M_{1}\right) \mathcal{P}^{\sigma_{2} \alpha_{2}}\left(M_{2}\right) \text {. }
\end{aligned}
$$

A complication arises when the pair consists of quarkonia with the same flavor. In this case, there exists an "exchange" diagram in which a quark from one gluon recombines with an antiquark from the other gluon and the remaining $q \bar{q}$ pair forms the second quarkonium. In order for this process to occur, the two quarks and antiquarks have to be all within the distance of order $1 / \mathrm{m}$. We thus cannot simply Taylor-expand the Wilson line separately in each gluon wave function as we have done above. Instead we must go back to the original expression and find the structure

$$
\begin{aligned}
& \operatorname{Tr}\left[U_{x_{1}} t^{c} U_{\boldsymbol{x}_{2}}^{\dagger} U_{\boldsymbol{y}_{1}} t^{c} U_{\boldsymbol{y}_{2}}^{\dagger}\right] \\
& =\frac{1}{2} \operatorname{Tr}\left[U_{\boldsymbol{x}_{2}}^{\dagger} U_{\boldsymbol{y}_{1}}\right] \operatorname{Tr}\left[U_{\boldsymbol{y}_{2}}^{\dagger} U_{\boldsymbol{x}_{1}}\right]-\frac{1}{2 N_{c}} \operatorname{Tr}\left[U_{x_{1}} U_{\boldsymbol{x}_{2}}^{\dagger} U_{\boldsymbol{y}_{1}} U_{\boldsymbol{y}_{2}}^{\dagger}\right],
\end{aligned}
$$

which is not associated with the WW gluon distribution. While this may seem a problem, it is intuitively clear that such a contribution is negligible in the limit of large quark mass. The probability to find all the four quarks and antiquarks within a small area of order $1 / \mathrm{m}^{2}$ is power suppressed compared to the "direct" contribution ${ }^{3}$ (see Fig. 1). However, this suppression is difficult to see in the NRQCD framework where to leading order one just multiplies the partonic cross section by the constant LDMEs. We think this is an artifact of the NRQCD approach, and the exchange diagram will be suppressed in a more complete treatment of the problem. Therefore, while (32) and similar results below are valid for the production of a different-flavor pair, strictly speaking, we think they can be also used for a same-flavor pair up to small corrections.

Before leaving this section, we should comment on the color-octet production mechanism. In NRQCD, quarkonia can be produced in a color-octet state [40]. It is well known that, in the collinear factorization framework, the color octet contribution actually dominates over the color-singlet contribution for $J / \psi$ production, and this is also the case for $\chi_{c}$ production at large transverse momentum [41]. In this paper, we instead focus on low transverse momentum quarkonia production $p_{T} \sim m$ where the color-singlet channel may actually dominate [41], although the NRQCD factorization in this region needs further investigation to clarify this issue. ${ }^{4}$ On the other hand, it has been found that $\eta_{c}$ production is always dominated by the color-singlet channel in the whole range of transverse momentum $[42,43]$. Moreover, in the $k_{T^{-}}$ factorization approach, $\chi_{c}$ production is clearly dominated by the color-singlet channel $[44,45]$. Indeed, in the $\chi_{J=1}$ production channel which will be our main focus, the subprocess $g g^{*} \rightarrow \chi_{1}^{\text {singlet }}$ is not forbidden by the LandauYang theorem because one of the gluons is off-shell in the $k_{T}$ and hybrid factorizations, although it is forbidden in the collinear factorization. As we are considering the forward production of quarkonia at low to moderate $p_{T}$, the use of $k_{T}$ or hybrid factorization is more appropriate. We thus concentrate on the color-singlet production mechanism in this paper, and leave the color-octet case for future work.

\section{HYBRID FACTORIZATION WITH DOUBLE SCATTERING}

\section{A. Double gluon PDF}

The 2 gluons-to-2 quarkonia production amplitude (31) is to be squared and convoluted with the double gluon distribution of the projectile proton with momentum $P_{P}$. In doing so, one has to be careful about the fact that the Lorentz indices $\left(\sigma_{1} \sigma_{2}\right)$ of the gluons in the amplitude and the complex-conjugate amplitude can in general be different. This forces us to consider the most general double gluon PDF [46]

\footnotetext{
${ }^{3}$ For studies of such contributions, the reader is referred to [38] for massive quarks, or [39] for an earlier study with massless quarks.

${ }^{4}$ From our derivation we find that the color-octet contribution is not sensitive to the WWW distribution that we are after. A cleaner approach to avoid this problem is to consider doubly diffractive events, $p p \rightarrow p M_{1} M_{2} p$ as in [31] The cross section is then quartic in the proton Wigner distribution.
} 


$$
\begin{aligned}
\mathcal{F}_{a_{1} a_{2}}\left(x_{1}, x_{2}, \boldsymbol{k}_{1}, \boldsymbol{k}_{2}, q\right)= & \frac{2}{x_{1} x_{2} P_{P}^{+}} \int d^{2} \boldsymbol{k}_{1} d^{2} \boldsymbol{k}_{2} \int \frac{d r^{-}}{2 \pi} \frac{d z^{-}}{2 \pi} \frac{d^{2} \boldsymbol{r}}{(2 \pi)^{2}} \frac{d^{2} z}{(2 \pi)^{2}} e^{i x_{1} P_{P}^{+} r^{-}+i x_{2} P_{P}^{+} z^{-}-i \boldsymbol{k}_{1} \cdot \boldsymbol{r}-i \boldsymbol{k}_{2} \cdot z} \\
& \times \int d y^{-} d^{2} \boldsymbol{y} e^{i \boldsymbol{q} \cdot \boldsymbol{y}}\left\langle P_{P}\left|\Pi_{a_{1}}^{i i^{\prime}} G^{+i^{\prime}}\left(-\frac{r}{2}\right) G^{+i}\left(\frac{r}{2}\right) \Pi_{a_{2}}^{j j^{\prime}} G^{+j^{\prime}}\left(y-\frac{z}{2}\right) G^{+j}\left(y+\frac{z}{2}\right)\right| P_{P}\right\rangle_{y^{+}=r^{+}=z^{+}=0},
\end{aligned}
$$

where $a_{1,2}=\{g, \Delta g, \delta g\}$ and $\Pi_{g}^{i i^{\prime}} \equiv \delta^{i i^{\prime}}, \Pi_{\Delta g}^{i i^{\prime}} \equiv i \epsilon^{i i^{\prime}}, \quad \Pi_{\delta g}^{i i^{\prime}} \equiv \tau^{i i^{\prime}, l l^{\prime}} \equiv \frac{1}{2}\left(\delta^{i l} i^{i^{\prime} l^{\prime}}+\delta^{i l^{\prime}} \delta^{i^{\prime} l}-\delta^{i i^{\prime}} \delta^{l l^{\prime}}\right)$ are respectively the unpolarized, longitudinally polarized and linearly polarized projectors. The momentum $\boldsymbol{q}$ is conjugate to the relative transverse coordinate of the two gluons, and its dependence cannot be completely eliminated [46].

\section{B. Polarization sum}

For unpolarized quarkonium production, we square the amplitude (31) and sum over quarkonium polarizations:

$$
\begin{aligned}
\mathcal{P}^{\sigma \alpha}\left({ }^{3} S_{0}\right) \mathcal{P}^{\sigma^{\prime} \alpha^{\prime}}\left({ }^{3} S_{0}\right) & =\left(K^{+}\right)^{2} \epsilon^{\sigma_{\perp} \alpha_{\perp}+-} \epsilon^{\sigma_{\perp}^{\prime} \alpha_{\perp}^{\prime}+-}, \\
\mathcal{P}^{\sigma \alpha}\left({ }^{3} P_{J}\right) \mathcal{P}^{\sigma^{\prime} \alpha^{\prime}}\left({ }^{3} P_{J}\right) & =\sum_{\text {pol }} \frac{\varepsilon_{(J) \rho \mu} \varepsilon_{(J) \rho^{\prime} \mu^{\prime}}}{m^{2}} \mathcal{P}^{\sigma \alpha \rho \mu} \mathcal{P}^{\sigma^{\prime} \alpha^{\prime} \rho^{\prime} \mu^{\prime},}
\end{aligned}
$$

where the indices in the complex conjugate amplitude are denoted with a prime. This can be evaluated explicitly using the following result for ${ }^{3} P_{J}$ polarization sums:

$$
\begin{aligned}
\varepsilon_{(0) \rho \mu} \varepsilon_{(0) \rho^{\prime} \mu^{\prime}}^{*} & =\frac{1}{3} \Pi_{\rho \mu} \Pi_{\rho^{\prime} \mu^{\prime}}, \\
\sum \varepsilon_{(1) \rho \mu} \varepsilon_{(1) \rho^{\prime} \mu^{\prime}}^{*} & =\frac{1}{2}\left(\Pi_{\rho \rho^{\prime}} \Pi_{\mu \mu^{\prime}}-\Pi_{\rho \mu^{\prime}} \Pi_{\rho^{\prime} \mu}\right), \\
\sum \varepsilon_{(2) \rho \mu} \varepsilon_{(2) \rho^{\prime} \mu^{\prime}}^{*} & =\frac{1}{2}\left(\Pi_{\rho \rho^{\prime}} \Pi_{\mu \mu^{\prime}}+\Pi_{\rho \mu^{\prime}} \Pi_{\rho^{\prime} \mu}\right)-\frac{1}{3} \Pi_{\rho \mu} \Pi_{\rho^{\prime} \mu^{\prime}},
\end{aligned}
$$

where

and the contractions

$$
\Pi_{\rho \mu} \equiv-g_{\mu \rho}+\frac{P_{\mu} P_{\rho}}{4 m^{2}}
$$

$$
\begin{aligned}
& \Pi_{\rho \mu} \Pi_{\rho^{\prime} \mu^{\prime}} \mathcal{K}^{\sigma \alpha \rho \mu} \mathcal{K}^{\sigma^{\prime} \alpha^{\prime} \rho^{\prime} \mu^{\prime}}=9\left(K^{+}\right)^{2} g_{\perp}^{\alpha \sigma} g_{\perp}^{\alpha^{\prime} \sigma^{\prime}} \\
& \Pi_{\rho \rho^{\prime}} \Pi_{\mu \mu^{\prime}} \mathcal{P}^{\sigma \alpha \rho \mu} \mathcal{P}^{\sigma^{\prime} \alpha^{\prime} \rho^{\prime} \mu^{\prime}}=\left(K^{+}\right)^{2}\left[2 g_{\perp}^{\alpha \alpha^{\prime}} g_{\perp}^{\sigma \sigma^{\prime}}+2 g_{\perp}^{\alpha \sigma^{\prime}} g_{\perp}^{\alpha^{\prime} \sigma}+g_{\perp}^{\alpha^{\prime} \sigma^{\prime}} g_{\perp}^{\alpha \sigma}-g_{\perp}^{\sigma \sigma^{\prime}} \frac{\left(K_{\perp}^{\alpha}-k_{\perp}^{\alpha}\right)\left(K_{\perp}^{\alpha^{\prime}}-\ell_{\perp}^{\alpha^{\prime}}\right)}{m^{2}}\right] \\
& \Pi_{\rho \mu^{\prime}} \Pi_{\rho^{\prime} \mu} \mathcal{P}^{\sigma \alpha \rho \mu} \mathcal{P}^{\sigma^{\prime} \alpha^{\prime} \rho^{\prime} \mu^{\prime}}=\left(K^{+}\right)^{2}\left[2 g_{\perp}^{\alpha \sigma^{\prime}} g_{\perp}^{\alpha^{\prime} \sigma}+2 g_{\perp}^{\alpha \alpha^{\prime}} g_{\perp}^{\sigma \sigma^{\prime}}+g_{\perp}^{\alpha \sigma} g_{\perp}^{\alpha^{\prime} \sigma^{\prime}}\right] .
\end{aligned}
$$

The result is

$$
\begin{aligned}
& \mathcal{P}^{\sigma \alpha}\left({ }^{3} P_{0}\right) \mathcal{P}^{\sigma^{\prime} \alpha^{\prime}}\left({ }^{3} P_{0}\right)=3 \frac{\left(K^{+}\right)^{2}}{m^{2}} g_{\perp}^{\alpha \sigma} g_{\perp}^{\alpha^{\prime} \sigma^{\prime}} \\
& \mathcal{P}^{\sigma \alpha}\left({ }^{3} P_{1}\right) \mathcal{P}^{\sigma^{\prime} \alpha^{\prime}}\left({ }^{3} P_{1}\right)=-\frac{\left(K^{+}\right)^{2}}{2 m^{4}}\left(K_{\perp}^{\alpha}-k_{\perp}^{\alpha}\right)\left(K_{\perp}^{\alpha^{\prime}}-\ell_{\perp}^{\alpha^{\prime}}\right) g_{\perp}^{\sigma \sigma^{\prime}} \\
& \mathcal{P}^{\sigma \alpha}\left({ }^{3} P_{2}\right) \mathcal{P}^{\sigma^{\prime} \alpha^{\prime}}\left({ }^{3} P_{2}\right)=\frac{2\left(K^{+}\right)^{2}}{m^{2}}\left[g_{\perp}^{\alpha \alpha^{\prime}} g_{\perp}^{\sigma \sigma^{\prime}}-g_{\perp}^{\alpha \sigma} g_{\perp}^{\alpha^{\prime} \sigma^{\prime}}+g_{\perp}^{\alpha \sigma^{\prime}} g_{\perp}^{\alpha^{\prime} \sigma}-\frac{\left(K_{\perp}^{\alpha}-k_{\perp}^{\alpha}\right)\left(K_{\perp}^{\alpha^{\prime}}-\ell_{\perp}^{\alpha^{\prime}}\right)}{4 m^{2}} g_{\perp}^{\sigma \sigma^{\prime}}\right] .
\end{aligned}
$$

Note that we wrote these quantities with distinct incoming gluon transverse momenta in the amplitude $k_{\perp}$ and in the complex conjugate amplitude $\ell_{\perp}$. This is required for the proper use of the double PDF (33).

\section{Full cross section}

We now have all the machinery to finally compute the full differential cross section. This is straightforward but tedious, as we have to consider all possible Lorentz index structures, namely, $\mathcal{G}_{1,2,3,4}$ from (3) and $a_{1,2}=\{g, \Delta g, \delta g\}$ in (33). The generic result is 


$$
\begin{aligned}
\frac{d \sigma\left(M_{1}, M_{2}\right)}{d Y_{1} d Y_{2} d^{2} \boldsymbol{\Delta} d^{2} \boldsymbol{K}}= & \frac{\alpha_{s}^{2}}{16 m_{1}^{5} m_{2}^{5} N_{c}^{4}\left(N_{c}^{2}-1\right)^{2}}\left\langle\mathcal{O}_{M_{1}}\left({ }^{2 S_{1}+1} L_{1 J_{1}}^{1}\right)\right\rangle\left\langle\mathcal{O}_{M_{2}}\left({ }^{2 S_{2}+1} L_{2 J_{2}}^{1}\right)\right\rangle \\
& \times x_{1} x_{2} \int d^{2} \boldsymbol{q}\left[\delta^{i i^{\prime}} \delta^{j j^{\prime}} \mathcal{F}_{g, g}\left(x_{1}, x_{2}, \boldsymbol{q}\right)-i \delta^{i i^{\prime}} \epsilon^{j j^{\prime}} \mathcal{F}_{g, \Delta g}\left(x_{1}, x_{2}, \boldsymbol{q}\right)+2 \delta^{i i^{\prime}} \tau^{j j^{\prime}, n n^{\prime}} \mathcal{F}_{g, \delta g}^{n n^{\prime}}\left(x_{1}, x_{2}, \boldsymbol{q}\right)\right. \\
& -i \epsilon^{i i^{\prime}} \delta^{j j^{\prime}} \mathcal{F}_{\Delta g, g}\left(x_{1}, x_{2}, \boldsymbol{q}\right)-\epsilon^{i i^{\prime}} \epsilon^{j j^{\prime}} \mathcal{F}_{\Delta g, \Delta g}\left(x_{1}, x_{2}, \boldsymbol{q}\right)-2 i \epsilon^{i i^{\prime}} \tau^{j j^{\prime}, n n^{\prime}} \mathcal{F}_{\Delta g, \delta g}^{n n^{\prime}}\left(x_{1}, x_{2}, \boldsymbol{q}\right) \\
& \left.+2 \delta^{j j^{\prime}} \tau^{i i^{\prime}, m m^{\prime}} \mathcal{F}_{\delta g, g}^{m m^{\prime}}\left(x_{1}, x_{2}, \boldsymbol{q}\right)-2 i \epsilon^{j j^{\prime}} \tau^{i i^{\prime}, m m^{\prime}} \mathcal{F}_{\delta g, \Delta g}^{m m^{\prime}}\left(x_{1}, x_{2}, \boldsymbol{q}\right)+4 \tau^{j j^{\prime}, n n^{\prime}} \tau^{i i^{\prime}, m m^{\prime}} \mathcal{F}_{\delta g, \delta g}^{m m^{\prime}, n n^{\prime}}\left(x_{1}, x_{2}, \boldsymbol{q}\right)\right] \\
& \times \Pi_{1}^{i i^{\prime}, k k^{\prime}}\left(M_{1}\right) \Pi_{2}^{j j^{\prime}, \ell \ell^{\prime}}\left(M_{2}\right) x \mathcal{G}^{k \ell}\left(\boldsymbol{K}-\frac{\boldsymbol{q}}{2}, \boldsymbol{\Delta}\right) x \mathcal{G}^{k^{\prime} \ell^{\prime} *}\left(\boldsymbol{K}+\frac{\boldsymbol{q}}{2}, \boldsymbol{\Delta}\right),
\end{aligned}
$$

where $x_{i} \equiv \frac{K_{i}^{+}}{P_{P}^{+}}, \boldsymbol{\Delta} \equiv \boldsymbol{K}_{1}+\boldsymbol{K}_{2}$, and from now on, $\boldsymbol{K} \equiv \frac{\boldsymbol{K}_{1}-\boldsymbol{K}_{2}}{2} . Y_{i}$ the rapidity of meson $M_{i}$, and the hard subparts $\Pi_{1,2}$ are given by

$$
\begin{aligned}
\Pi_{1}^{i i^{\prime}, k k^{\prime}}(\eta) & =\delta^{i i^{\prime}} \delta^{k k^{\prime}}-\delta^{i k^{\prime}} \delta^{i^{\prime} k}, \\
\Pi_{1}^{i i^{\prime}, k k^{\prime}}\left(\chi_{0}\right) & =3 \frac{\delta^{i k} \delta^{i^{\prime} k^{\prime}}}{m_{1}^{2}}, \\
\Pi_{1}^{i i^{\prime}, k k^{\prime}}\left(\chi_{1}\right) & =\frac{\left(\boldsymbol{K}^{k}+\frac{\Delta^{k}-\boldsymbol{q}^{k}}{2}\right)\left(\boldsymbol{K}^{k^{\prime}}+\frac{\Delta^{k^{\prime}}+\boldsymbol{q}^{k^{\prime}}}{2}\right)}{2 m_{1}^{4}} \delta^{i i^{\prime}}, \\
\Pi_{1}^{i i^{\prime}, k k^{\prime}}\left(\chi_{2}\right) & =\frac{2}{m_{1}^{2}}\left[\delta^{i i^{\prime}} \delta^{k k^{\prime}}-\delta^{i k} \delta^{i^{\prime} k^{\prime}}+\delta^{i^{\prime} k} \delta^{i k^{\prime}}+\frac{\left(\boldsymbol{K}^{k}+\frac{\Delta^{k}-\boldsymbol{q}^{k}}{2}\right)\left(\boldsymbol{K}^{k^{\prime}}+\frac{\Delta^{k^{\prime}}+\boldsymbol{q}^{k^{\prime}}}{2}\right)}{4 m_{1}^{2}} \delta^{i i^{\prime}}\right],
\end{aligned}
$$

for meson 1 and

$$
\begin{aligned}
\Pi_{2}^{j j^{\prime}, \ell \ell^{\prime}}(\eta) & =\delta^{j j^{\prime}} \delta^{\ell \ell^{\prime}}-\delta^{j \ell^{\prime}} \delta^{j^{\prime} \ell}, \\
\Pi_{2}^{j j^{\prime}, \ell \ell^{\prime}}\left(\chi_{0}\right) & =3 \frac{\delta^{j \ell} \delta^{j^{\prime} \ell^{\prime}}}{m_{2}^{2}}, \\
\Pi_{2}^{j j^{\prime}, \ell \ell^{\prime}}\left(\chi_{1}\right) & =\frac{\left(\boldsymbol{K}^{\ell}-\frac{\boldsymbol{\Delta}^{\ell}-\boldsymbol{q}^{\ell}}{2}\right)\left(\boldsymbol{K}^{\ell^{\prime}}-\frac{\boldsymbol{\Delta}^{\ell^{\prime}}+\boldsymbol{q}^{\ell^{\prime}}}{2}\right)}{2 m_{2}^{4}} \delta^{j j^{\prime}}, \\
\Pi_{2}^{j j^{\prime}, \ell \ell^{\prime}}\left(\chi_{2}\right) & =\frac{2}{m_{2}^{2}}\left[\delta^{j j^{\prime}} \delta^{\ell \ell^{\prime}}-\delta^{j \ell} \delta^{j^{\prime} \ell^{\prime}}+\delta^{j^{\prime} \ell} \delta^{j \ell^{\prime}}+\frac{\left(\boldsymbol{K}^{\ell}-\frac{\boldsymbol{\Delta}^{\ell}-\boldsymbol{q}^{\ell}}{2}\right)\left(\boldsymbol{K}^{\ell^{\prime}}-\frac{\boldsymbol{\Delta}^{\ell^{\prime}}+\boldsymbol{q}^{\ell^{\prime}}}{2}\right)}{4 m_{2}^{2}} \delta^{j j^{\prime}}\right],
\end{aligned}
$$

for meson 2. Interestingly, the intrinsic momentum $\boldsymbol{q}$ of the double PDF enters the argument of the WW GTMD. Physically, $\boldsymbol{q}$ is conjugate to the relative distance of the two quarkonia. More precisely, it is conjugate to $\frac{\boldsymbol{b}_{1}+\boldsymbol{b}_{1}^{\prime}}{2}-$ $\frac{\boldsymbol{b}_{2}+\boldsymbol{b}_{2}^{\prime}}{2}$ where $\boldsymbol{b}_{1,2}$ is as in (31) and $\boldsymbol{b}_{1,2}^{\prime}$ are the corresponding coordinates in the complex-conjugate amplitude.

\section{EXPLICIT CROSS SECTIONS}

In the following, we will assume that $|\boldsymbol{K}| \gg|\boldsymbol{q}|$. The dependence on $\boldsymbol{q}$ is then completely absorbed in the projectile double PDF. We will thus define the integrated double PDFs

$$
\mathcal{F}_{a_{1}, a_{2}}\left(x_{1}, x_{2}\right) \equiv \int d^{2} \boldsymbol{q} \mathcal{F}_{a_{1}, a_{2}}\left(x_{1}, x_{2}, \boldsymbol{q}\right) .
$$

At this point, we can already cancel some contributions. Indeed the symmetry and tracelessness properties of the $\left(a_{1}=g, a_{2}=\delta g\right)$ double PDF allows one to write it as:

$$
\mathcal{F}_{g, \delta g}^{m n}\left(x_{1}, x_{2}, \boldsymbol{q}\right)=\left(\frac{\boldsymbol{q}^{m} \boldsymbol{q}^{n}}{\boldsymbol{q}^{2}}-\frac{\delta^{m n}}{2}\right) \frac{\boldsymbol{q}^{2}}{M_{P}^{2}} \mathcal{H}_{g, \delta g}\left(x_{1}, x_{2}, \boldsymbol{q}^{2}\right),
$$

where $M_{P}$ is the projectile's mass. It is then easy to show that the integral given in (43) cancels for this integrated double PDF. Similarly, we can cancel the $\left(a_{1}=\delta g, a_{2}=g\right)$ integrated double PDF.

It will also be useful to write the $\left(a_{1}, a_{2}\right)=(\delta g, \delta g)$ integrated double PDF as

$$
\begin{aligned}
\mathcal{F}_{\delta g, \delta g}^{m m^{\prime}, n n^{\prime}}\left(x_{1}, x_{2}\right) \equiv & \frac{1}{2}\left(\delta^{m n} \delta^{m^{\prime} n^{\prime}}+\delta^{m n^{\prime}} \delta^{m^{\prime} n}-\delta^{m m^{\prime}} \delta^{n n^{\prime}}\right) \\
& \times \mathcal{H}_{\delta g, \delta g}\left(x_{1}, x_{2}\right)
\end{aligned}
$$




\section{A. $\chi_{1} \chi_{1}$ cross section}

We find that the most concise formula is obtained for the double $\chi_{J=1}$ production. It is given by

$$
\frac{d \sigma\left(\chi_{f_{1} 1}, \chi_{f_{2} 1}\right)}{d Y_{1} d Y_{2} d^{2} \boldsymbol{\Delta} d^{2} \boldsymbol{K}}=\frac{x_{1} x_{2} \mathcal{F}_{g, g}\left(x_{1}, x_{2}\right)}{64 m_{1}^{9} m_{2}^{9} N_{c}^{4}\left(N_{c}^{2}-1\right)^{2}} \alpha_{s}^{4}\left\langle\mathcal{O}_{\chi_{f_{1} 1}}\left({ }^{3} P_{1}^{1}\right)\right\rangle\left\langle\mathcal{O}_{\chi_{f_{2} 1}}\left({ }^{3} P_{1}^{1}\right)\right\rangle\left|\left(\boldsymbol{K}^{i}+\frac{\boldsymbol{\Delta}^{i}}{2}\right)\left(\boldsymbol{K}^{j}-\frac{\boldsymbol{\Delta}^{j}}{2}\right) x \mathcal{G}^{i j}(\boldsymbol{K}, \boldsymbol{\Delta})\right|^{2},
$$

with $f_{1,2}$ the meson flavors. To check that the right-hand side has the correct dimensions, we note that $\operatorname{dim} \mathcal{G}=-2$, $\operatorname{dim} \mathcal{F}=2$, and $\operatorname{dim}\left\langle O_{\chi}\right\rangle=5\left(\operatorname{dim}\left\langle O_{\eta}\right\rangle=3\right)$. Explicitly, the second line of (46) reads

$$
\begin{aligned}
\left|\left(\boldsymbol{K}^{i}+\frac{\boldsymbol{\Delta}^{i}}{2}\right)\left(\boldsymbol{K}^{j}-\frac{\boldsymbol{\Delta}^{j}}{2}\right) x \mathcal{G}^{i j}(\boldsymbol{K}, \boldsymbol{\Delta})\right|^{2} & =\boldsymbol{K}^{4} \mid\left(1-\frac{\boldsymbol{\Delta}^{2}}{4 \boldsymbol{K}^{2}}\right) \mathcal{G}_{1}+\left(1-\frac{(\boldsymbol{K} \cdot \boldsymbol{\Delta})^{2}}{2 \boldsymbol{K}^{4}}+\frac{\boldsymbol{\Delta}^{2}}{4 \boldsymbol{K}^{2}}\right) \frac{\boldsymbol{K}^{2}}{2 M^{2}} \mathcal{G}_{2} \\
& -\left(\frac{\boldsymbol{\Delta}^{2}}{2 M^{2}}-\frac{(\boldsymbol{K} \cdot \boldsymbol{\Delta})^{2}}{\boldsymbol{K}^{2} M^{2}}+\frac{\boldsymbol{\Delta}^{4}}{8 \boldsymbol{K}^{2} M^{2}}\right) \mathcal{G}_{3}-\left.\left(\frac{\boldsymbol{\Delta}^{2}}{M^{2}}-\frac{(\boldsymbol{K} \cdot \boldsymbol{\Delta})^{2}}{\boldsymbol{K}^{2} M^{2}}\right) \mathcal{G}_{4}\right|^{2} \\
& \approx \boldsymbol{K}^{4}\left(\mathcal{G}_{1}+\frac{\boldsymbol{K}^{2}}{2 M^{2}} \mathcal{G}_{2}\right)^{2}
\end{aligned}
$$

where the last line is obtained by assuming $|\boldsymbol{K}| \gg|\boldsymbol{\Delta}|$. Remarkably, the cross section is directly proportional to the WW GTMD squared, without any convolution in momentum.

\section{B. All averaged cross sections}

We will now present all the full cross sections for our $\left(M_{1}, M_{2}\right)$ process, for $M_{i} \in\left\{\eta, \chi_{0}, \chi_{1}, \chi_{2}\right\}$. In order to get rid of the contributions with a longitudinally polarized double PDF, we will take the average with respect to the angle between $\boldsymbol{K}$ and $\boldsymbol{\Delta}$. This allows to keep only $\mathcal{F}_{g, g}\left(x_{1}, x_{2}\right)$ and $\mathcal{H}_{\delta g, \delta g}\left(x_{1}, x_{2}\right)$ as the nonperturbative distributions on the projectile side, since our goal is to focus rather on the nonperturbative effects on the target side.

\section{1. $\left(\chi_{1} \chi_{1}\right)$}

$$
\begin{aligned}
\int_{0}^{2 \pi} \frac{d \phi}{2 \pi} \frac{d \sigma\left(\chi_{f_{1} 1}, \chi_{f_{2} 1}\right)}{d Y_{1} d Y_{2} d^{2} \boldsymbol{K} d \boldsymbol{\Delta}^{2}}= & \frac{\alpha_{s}^{4} x^{2} \boldsymbol{K}^{4}}{32 m_{1}^{9} m_{2}^{9} N_{c}^{4}\left(N_{c}^{2}-1\right)^{2}} x_{1} x_{2} \mathcal{F}_{g, g}\left(x_{1}, x_{2}\right)\left\langle\mathcal{O}_{\chi_{f_{1} 1}}\left({ }^{3} P_{1}^{1}\right)\right\rangle\left\langle\mathcal{O}_{\chi_{f_{2} 1}}\left({ }^{3} P_{1}^{1}\right)\right\rangle \\
& \times\left[\left(\mathcal{G}_{1}+\frac{\boldsymbol{K}^{2}}{2 M^{2}} \mathcal{G}_{2}\right)^{2}-\frac{\boldsymbol{\Delta}^{2}}{2 \boldsymbol{K}^{2}}\left(\mathcal{G}_{1}+\frac{\boldsymbol{K}^{2}}{2 M^{2}} \mathcal{G}_{2}\right)\left(\mathcal{G}_{1}+2 \frac{\boldsymbol{K}^{2}}{M^{2}} \mathcal{G}_{4}\right)\right]
\end{aligned}
$$

\section{2. $\left(\chi_{1} \chi_{0}\right)$}

$$
\begin{aligned}
\int_{0}^{2 \pi} \frac{d \phi}{2 \pi} \frac{d \sigma\left(\chi_{1}, \chi_{0}\right)}{d Y_{1} d Y_{2} d^{2} \boldsymbol{K} d \boldsymbol{\Delta}^{2}}= & \frac{3 \alpha_{s}^{4} x^{2} \boldsymbol{K}^{2}}{32 m_{1}^{9} m_{2}^{7} N_{c}^{4}\left(N_{c}^{2}-1\right)^{2}} x_{1} x_{2} \mathcal{F}_{g, g}\left(x_{1}, x_{2}\right)\left\langle\mathcal{O}_{\chi_{1}}\left({ }^{3} P_{1}^{1}\right)\right\rangle\left\langle\mathcal{O}_{\chi_{0}}\left({ }^{3} P_{0}^{1}\right)\right\rangle \\
& \times\left[\left(\mathcal{G}_{1}+\frac{\boldsymbol{K}^{2}}{2 M^{2}} \mathcal{G}_{2}\right)^{2}+\frac{\boldsymbol{\Delta}^{2}}{4 \boldsymbol{K}^{2}}\left(\mathcal{G}_{1}^{2}+\frac{\boldsymbol{K}^{4}}{4 M^{4}}\left(\mathcal{G}_{2}^{2}-8 \mathcal{G}_{2} \mathcal{G}_{4}+8 \mathcal{G}_{4}^{2}\right)\right)\right]
\end{aligned}
$$

\section{3. $\left(\chi_{1} \chi_{2}\right)$}

$$
\begin{aligned}
\int_{0}^{2 \pi} \frac{d \phi}{2 \pi} \frac{d \sigma\left(\chi_{1}, \chi_{2}\right)}{d Y_{1} d Y_{2} d^{2} \boldsymbol{K} d \boldsymbol{\Delta}^{2}}= & \frac{\alpha_{s}^{4} \boldsymbol{K}^{2} x^{2}}{8 m_{1}^{7} m_{2}^{7} N_{c}^{4}\left(N_{c}^{2}-1\right)^{2}}\left\langle\mathcal{O}_{\chi_{1}}\left({ }^{3} P_{1}^{1}\right)\right\rangle\left\langle\mathcal{O}_{\chi_{2}}\left({ }^{3} P_{2}^{1}\right)\right\rangle x_{1} x_{2} \mathcal{F}_{g, g}\left(x_{1}, x_{2}\right)\left\{\left(1+\frac{\boldsymbol{K}^{2}}{4 m_{2}^{2}}\right)\left(\mathcal{G}_{1}+\frac{\boldsymbol{K}^{2}}{2 M^{2}} \mathcal{G}_{2}\right)^{2}\right. \\
& \left.+\frac{\boldsymbol{\Delta}^{2}}{4 \boldsymbol{K}^{2}}\left[\left(1+\frac{\boldsymbol{K}^{2}}{m_{2}^{2}}\right) \mathcal{G}_{1}^{2}+\frac{3 \boldsymbol{K}^{4}}{4 m_{2}^{2} M^{2}} \mathcal{G}_{1} \mathcal{G}_{2}+\frac{\boldsymbol{K}^{4}}{4 M^{4}}\left(1+\frac{\boldsymbol{K}^{2}}{2 m_{2}^{2}}\right) \mathcal{G}_{2}^{2}-2 \frac{\boldsymbol{K}^{4}}{\boldsymbol{M}^{4}}\left(\mathcal{G}_{2}-\mathcal{G}_{4}\right) \mathcal{G}_{4}\right]\right\}
\end{aligned}
$$




\section{4. $\left(\chi_{1} \eta\right)$}

$$
\begin{aligned}
& \frac{1}{2 \pi} \int_{0}^{2 \pi} d \phi \frac{d \sigma\left(\chi_{1}, \eta\right)}{d Y_{1} d Y_{2} d \boldsymbol{\Delta}^{2} d^{2} \boldsymbol{K}}=\frac{\alpha_{s}^{4} x^{2} \boldsymbol{K}^{2}}{32 m_{1}^{7} m_{2}^{5} N_{c}^{4}\left(N_{c}^{2}-1\right)^{2}} x_{1} x_{2} \mathcal{F}_{g, g}\left(x_{1}, x_{2}\right)\left\langle\mathcal{O}_{\chi_{1}}\left({ }^{3} P_{1}^{1}\right)\right\rangle\left\langle\mathcal{O}_{\eta}\left({ }^{1} S_{0}^{1}\right)\right\rangle \\
& \times\left[\left(\mathcal{G}_{1}+\frac{\boldsymbol{K}^{2}}{2 M^{2}} \mathcal{G}_{2}\right)^{2}+\frac{\boldsymbol{\Delta}^{2}}{4 \boldsymbol{K}^{2}}\left(\mathcal{G}_{1}^{2}+\frac{\boldsymbol{K}^{4}}{4 M^{4}}\left(\mathcal{G}_{2}^{2}-8 \mathcal{G}_{2} \mathcal{G}_{4}+8 \mathcal{G}_{4}^{2}\right)\right)\right] \\
& \text { 5. }\left(\chi_{0} \chi_{0}\right) \\
& \int_{0}^{2 \pi} \frac{d \phi}{2 \pi} \frac{d \sigma\left(\chi_{f_{1} 0}, \chi_{f_{2} 0}\right)}{d Y_{1} d Y_{2} d^{2} \boldsymbol{K} d \boldsymbol{\Delta}^{2}}=\frac{9 \alpha_{s}^{4} x^{2}}{16 m_{1}^{7} m_{2}^{7} N_{c}^{4}\left(N_{c}^{2}-1\right)^{2}}\left\langle\mathcal{O}_{\chi_{f_{1} 0}}\left({ }^{3} P_{0}^{1}\right)\right\rangle\left\langle\mathcal{O}_{\chi_{f_{2} 0}}\left({ }^{3} P_{0}^{1}\right)\right\rangle\left\{x_{1} x_{2} \mathcal{F}_{g, g}\left(x_{1}, x_{2}\right)\left(\mathcal{G}_{1}^{2}+\frac{\boldsymbol{K}^{4}}{4 M^{4}} \mathcal{G}_{2}^{2}+\frac{\boldsymbol{K}^{2} \boldsymbol{\Delta}^{2}}{2 M^{4}} \mathcal{G}_{4}^{2}\right)\right. \\
& \left.+4 x_{1} x_{2} \mathcal{H}_{\delta g, \delta g}\left(x_{1}, x_{2}\right)\left[\mathcal{G}_{1}^{2}-2 \frac{\Delta^{2}}{M^{2}}\left(\mathcal{G}_{1} \mathcal{G}_{3}-\frac{\boldsymbol{K}^{2}}{4 M^{2}} \mathcal{G}_{4}^{2}\right)\right]\right\} \\
& \text { 6. }\left(\chi_{0} \chi_{2}\right)
\end{aligned}
$$

$$
\begin{aligned}
\int_{0}^{2 \pi} \frac{d \phi}{2 \pi} \frac{d \sigma\left(\chi_{0}, \chi_{2}\right)}{d Y_{1} d Y_{2} d^{2} \boldsymbol{K} d \boldsymbol{\Delta}^{2}}= & \frac{3 \alpha_{s}^{4} x^{2}}{16 m_{1}^{7} m_{2}^{7} N_{c}^{4}\left(N_{c}^{2}-1\right)^{2}} x_{1} x_{2} \mathcal{F}_{g, g}\left(x_{1}, x_{2}\right)\left\langle\mathcal{O}_{\chi_{0}}\left({ }^{3} P_{0}^{1}\right)\right\rangle\left\langle\mathcal{O}_{\chi_{2}}\left({ }^{3} P_{2}^{1}\right)\right\rangle \\
& \times\left[4 \mathcal{G}_{1}^{2}+\frac{\boldsymbol{K}^{4}}{M^{4}} \mathcal{G}_{2}^{2}+\frac{\boldsymbol{K}^{2}}{2 m_{2}^{2}}\left(\mathcal{G}_{1}+\frac{\boldsymbol{K}^{2}}{2 M^{2}} \mathcal{G}_{2}\right)^{2}\right. \\
& \left.+\frac{\boldsymbol{\Delta}^{2}}{8 M^{2}}\left(\frac{M^{2}}{m_{2}^{2}} \mathcal{G}_{1}^{2}+16 \frac{\boldsymbol{K}^{2}}{M^{2}} \mathcal{G}_{4}^{2}+\frac{\boldsymbol{K}^{4}}{4 m_{2}^{2} M^{2}}\left(\mathcal{G}_{2}^{2}+8 \mathcal{G}_{2} \mathcal{G}_{4}+8 \mathcal{G}_{4}^{2}\right)\right)\right] \\
\int_{0}^{2 \pi} \frac{d \phi}{2 \pi} \frac{d \sigma\left(\chi_{0}, \eta\right)}{d Y_{1} d Y_{2} d^{2} \boldsymbol{K} d \boldsymbol{\Delta}^{2}}= & \frac{3 \alpha_{0}^{4} x^{2}}{16 m_{1}^{7} m_{2}^{5} N_{c}^{4}\left(N_{c}^{2}-1\right)^{2}}\left\langle\mathcal{O}_{\chi_{0}}\left({ }^{3} P_{0}^{1}\right)\right\rangle\left\langle\mathcal{O}_{\eta}\left({ }^{1} S_{0}^{1}\right)\right\rangle\left\{x_{1} x_{2} \mathcal{F}_{g, g}\left(x_{1}, x_{2}\right)\left(\mathcal{G}_{1}^{2}+\frac{\boldsymbol{K}^{4}}{4 M^{4}} \mathcal{G}_{2}^{2}+\frac{\boldsymbol{K}^{2} \boldsymbol{\Delta}^{2}}{2 M^{4}} \mathcal{G}_{4}^{2}\right)\right. \\
& \left.-4 x_{1} x_{2} \mathcal{H}_{\delta g, \delta g}\left(x_{1}, x_{2}\right)\left[\mathcal{G}_{1}^{2}-2 \frac{\Delta^{2}}{M^{2}}\left(\mathcal{G}_{1} \mathcal{G}_{3}-\frac{\boldsymbol{K}^{2}}{4 M^{2}} \mathcal{G}_{4}^{2}\right)\right]\right\}
\end{aligned}
$$

8. $\left(\chi_{2} \chi_{2}\right)$

$$
\begin{aligned}
\int_{0}^{2 \pi} \frac{d \phi}{2 \pi} \frac{d \sigma\left(\chi_{f_{1} 2}, \chi_{f_{2} 2}\right)}{d Y_{1} d Y_{2} d^{2} \boldsymbol{K} d \boldsymbol{\Delta}^{2}}= & \frac{\alpha_{s}^{4} x^{2}}{8 m_{1}^{7} m_{2}^{7} N_{c}^{4}\left(N_{c}^{2}-1\right)^{2}} x_{1} x_{2} \mathcal{F}_{g, g}\left(x_{1}, x_{2}\right)\left\langle\mathcal{O}_{\chi_{f_{1} 2}}\left({ }^{3} P_{2}^{1}\right)\right\rangle\left\langle\mathcal{O}_{\chi_{f_{2} 2}}\left({ }^{3} P_{2}^{1}\right)\right\rangle \\
& \times\left\{8\left(\mathcal{G}_{1}^{2}+\frac{\boldsymbol{K}^{4}}{4 M^{4}} \mathcal{G}_{2}^{2}\right)+\left(\frac{\boldsymbol{K}^{2}}{m_{1}^{2}}+\frac{\boldsymbol{K}^{2}}{m_{2}^{2}}+\frac{\boldsymbol{K}^{4}}{4 m_{1}^{2} m_{2}^{2}}\right)\left(\mathcal{G}_{1}+\frac{\boldsymbol{K}^{2}}{2 M^{2}} \mathcal{G}_{2}\right)^{2}\right. \\
& +\frac{\boldsymbol{\Delta}^{2}}{32 m_{1}^{2} m_{2}^{2}}\left[8 \mathcal{G}_{1}^{2}\left(\boldsymbol{K}^{2}+m_{1}^{2}+m_{2}^{2}\right)+6 \boldsymbol{K}^{2} \frac{\boldsymbol{K}^{2}}{M^{2}} \mathcal{G}_{1} \mathcal{G}_{2}+\frac{\boldsymbol{K}^{4}}{M^{4}}\left(\boldsymbol{K}^{2}+2 m_{1}^{2}+2 m_{2}^{2}\right) \mathcal{G}_{2}^{2}\right] \\
& \left.+\frac{\boldsymbol{\Delta}^{2}}{2 m_{1}^{2} m_{2}^{2}} \frac{\boldsymbol{K}^{2}}{\boldsymbol{M}^{2}}\left[\left(m_{1}^{2}-m_{2}^{2}\right) \frac{\boldsymbol{K}^{2}}{\boldsymbol{M}^{2}} \mathcal{G}_{2} \mathcal{G}_{4}+\left(\frac{\boldsymbol{K}^{2} m_{1}^{2}}{M^{2}}+\frac{\boldsymbol{K}^{2} m_{2}^{2}}{M^{2}}+8 \frac{m_{1}^{2} m_{2}^{2}}{M^{2}}\right) \mathcal{G}_{4}^{2}\right]\right\}
\end{aligned}
$$

9. $\left(\chi_{2} \boldsymbol{\eta}\right)$

$$
\begin{aligned}
\int_{0}^{2 \pi} \frac{d \phi}{2 \pi} \frac{d \sigma\left(\chi_{2}, \eta\right)}{d Y_{1} d Y_{2} d^{2} \boldsymbol{K} d \boldsymbol{\Delta}^{2}}= & \frac{\alpha_{s}^{4} x^{2}}{16 m_{1}^{7} m_{2}^{5} N_{c}^{4}\left(N_{c}^{2}-1\right)^{2}}\left\langle\mathcal{O}_{\chi_{2}}\left({ }^{3} P_{2}^{1}\right)\right\rangle\left\langle\mathcal{O}_{\eta}\left({ }^{1} S_{0}^{1}\right)\right\rangle \\
& \times x_{1} x_{2} \mathcal{F}_{g, g}\left(x_{1}, x_{2}\right)\left[4\left(\mathcal{G}_{1}^{2}+\frac{\boldsymbol{K}^{4}}{4 M^{4}} \mathcal{G}_{2}^{2}\right)+\frac{\boldsymbol{K}^{2}}{2 m_{1}^{2}}\left(\mathcal{G}_{1}+\frac{\boldsymbol{K}^{2}}{2 M^{2}} \mathcal{G}_{2}\right)^{2}\right. \\
& \left.+\frac{\boldsymbol{\Delta}^{2}}{8 m_{1}^{2}}\left(\mathcal{G}_{1}^{2}+\frac{\boldsymbol{K}^{4}}{4 M^{4}}\left(\mathcal{G}_{2}^{2}-8 \mathcal{G}_{2} \mathcal{G}_{4}+8 \mathcal{G}_{4}^{2}\right)+16 \frac{\boldsymbol{K}^{2}}{M^{2}} \frac{m_{1}^{2}}{M^{2}} \mathcal{G}_{4}^{2}\right)\right]
\end{aligned}
$$




$$
\begin{aligned}
\int_{0}^{2 \pi} \frac{d \phi}{2 \pi} \frac{d \sigma\left(\eta_{f_{1}}, \eta_{f_{2}}\right)}{d Y_{1} d Y_{2} d^{2} \boldsymbol{K} d \boldsymbol{\Delta}^{2}}= & \frac{\alpha_{s}^{4} x^{2}}{16 m_{1}^{5} m_{2}^{5} N_{c}^{4}\left(N_{c}^{2}-1\right)^{2}}\left\langle\mathcal{O}_{\eta_{f_{1}}}\left({ }^{1} S_{0}^{1}\right)\right\rangle\left\langle\mathcal{O}_{\eta_{f_{2}}}\left({ }^{1} S_{0}^{1}\right)\right\rangle\left\{x_{1} x_{2} \mathcal{F}_{g, g}\left(x_{1}, x_{2}\right)\left(\mathcal{G}_{1}^{2}+\frac{\boldsymbol{K}^{4}}{4 M^{4}} \mathcal{G}_{2}^{2}+\frac{\boldsymbol{K}^{2} \boldsymbol{\Delta}^{2}}{2 M^{4}} \mathcal{G}_{4}^{2}\right)\right. \\
& \left.+4 x_{1} x_{2} \mathcal{H}_{\delta g, \delta g}\left(x_{1}, x_{2}\right)\left[\mathcal{G}_{1}^{2}-2 \frac{\boldsymbol{\Delta}^{2}}{M^{2}}\left(\mathcal{G}_{1} \mathcal{G}_{3}-\frac{\boldsymbol{K}^{2}}{4 M^{2}} \mathcal{G}_{4}^{2}\right)\right]\right\}
\end{aligned}
$$

\section{CONCLUSIONS}

In summary, in this paper we derive the diffractive double quarkonia production in $p p$ and $p A$ collisions to probe the WW gluon GTMDs. In particular, we applied the double parton scattering mechanism from the projectile, where the two gluons scatter off the nucleon/nucleus target diffractively to produce the final state two quarkonium states. The amplitudes are found to be sensitive to the WW gluon GTMDs.

More importantly, we found that the differential cross sections can be much simplified if we integrate out the transverse momenta of the gluons from the projectile. The explicit expressions show that the cross sections can be written as squared of the WW gluon GTMDs from the target.

Our result is a first example of direct access to the WW gluon GTMDs in hard diffractive processes. Experimentally, this process may be a challenge to measure. We hope that our derivations will stimulate further theoretical developments to explore the physics of GTMD and possibility to measure them in experiments.

\section{ACKNOWLEDGMENTS}

This work is supported by the National Science Center, Poland, Grant No. 2015/17/B/ST2/01838. It is also partially supported by the Natural Science Foundation of China (NSFC) under Grant No. 11575070 and by the U.S. Department of Energy, Office of Science, Office of Nuclear Physics, under Contract No. DE-AC02-05CH11231.

\section{APPENDIX: GLUON TO ${ }^{1} S_{0}$ AND ${ }^{3} P_{J}$ WAVES TRANSITION IN THE HYBRID FORMALISM}

For completeness, here we write down the cross section for the single-inclusive quarkonium production $g p \rightarrow M X$ for ${ }^{1} S_{0}$ and ${ }^{3} P_{J}$ waves. Squaring (29) and using the projectors (34)-(38), we find

$$
\begin{aligned}
\frac{d \sigma_{g}(M)}{d K^{+} d^{2} \boldsymbol{K}}= & \frac{\alpha_{s}}{2 m^{5} N_{c}\left(N_{c}^{2}-1\right)} \delta\left(K^{+}-k^{+}\right) \\
& \times \int \frac{d^{2} \boldsymbol{b} d^{2} \boldsymbol{b}^{\prime}}{(2 \pi)^{2}} e^{i \boldsymbol{K} \cdot\left(\boldsymbol{b}^{\prime}-\boldsymbol{b}\right)} \\
& \times\left\langle\frac{1}{N_{c}} \operatorname{Tr}\left[U_{\boldsymbol{b}}^{\dagger}\left(\partial_{i} U_{\boldsymbol{b}}\right) U_{\boldsymbol{b}^{\prime}}^{\dagger}\left(\partial_{j} U_{\boldsymbol{b}^{\prime}}\right)\right]\right\rangle \\
& \times \varphi^{i j}\left({ }^{2 S+1} L_{J}\right)\left\langle\mathcal{O}_{M}\left({ }^{2 S+1} L_{J}^{1}\right)\right)^{\frac{1}{2}}
\end{aligned}
$$

where

$$
\begin{aligned}
& \varphi^{i j}\left({ }^{1} S_{0}\right)=\delta^{i j}, \quad \varphi^{i j}\left({ }^{3} P_{0}\right)=\frac{3 \delta^{i j}}{m^{2}}, \quad \varphi^{i j}\left({ }^{3} P_{1}\right)=\frac{K^{i} K^{j}}{m^{4}}, \\
& \varphi^{i j}\left({ }^{3} P_{2}\right)=\frac{4}{m^{2}}\left(\delta^{i j}+\frac{K^{i} K^{j}}{4 m^{2}}\right),
\end{aligned}
$$

and the brackets denote the forward matrix element $\frac{\langle P|\ldots| P\rangle}{\langle P \mid P\rangle}$, or equivalently the CGC averaging. Taking the case where $M=J / \psi$ and taking the small-dipole limit in the collinear limit of results described in [34] shows full compatibility of the present results with previous calculations.
[1] X. d. Ji, Viewing the Proton through "Color" Filters, Phys. Rev. Lett. 91, 062001 (2003).

[2] A. V. Belitsky, X. Ji, and F. Yuan, Quark imaging in the proton via quantum phase space distributions, Phys. Rev. D 69, 074014 (2004).

[3] C. Lorce and B. Pasquini, Quark Wigner distributions and orbital angular momentum, Phys. Rev. D 84, 014015 (2011).
[4] S. Meissner, A. Metz, and M. Schlegel, Generalized parton correlation functions for a spin-1/2 hadron, J. High Energy Phys. 08 (2009) 056.

[5] C. Lorce and B. Pasquini, Structure analysis of the generalized correlator of quark and gluon for a spin-1/2 target, J. High Energy Phys. 09 (2013) 138.

[6] M. G. Echevarria, A. Idilbi, K. Kanazawa, C. Lorc, A. Metz, B. Pasquini, and M. Schlegel, Proper definition and 
evolution of generalized transverse momentum dependent distributions, Phys. Lett. B 759, 336 (2016).

[7] P. J. Mulders and J. Rodrigues, Transverse momentum dependence in gluon distribution and fragmentation functions, Phys. Rev. D 63, 094021 (2001).

[8] C. J. Bomhof, P. J. Mulders, and F. Pijlman, The construction of gauge-links in arbitrary hard processes, Eur. Phys. J. C 47, 147 (2006).

[9] B. W. Xiao and F. Yuan, Non-Universality of Transverse Momentum Dependent Parton Distributions at Small-x, Phys. Rev. Lett. 105, 062001 (2010).

[10] F. Dominguez, B. W. Xiao, and F. Yuan, $k_{t}$-Factorization for Hard Processes in Nuclei, Phys. Rev. Lett. 106, 022301 (2011).

[11] F. Dominguez, C. Marquet, B. W. Xiao, and F. Yuan, Universality of unintegrated gluon distributions at small X, Phys. Rev. D 83, 105005 (2011).

[12] Y. Hatta, B. W. Xiao, and F. Yuan, Probing the Small-x Gluon Tomography in Correlated Hard Diffractive Dijet Production in Deep Inelastic Scattering, Phys. Rev. Lett. 116, 202301 (2016).

[13] D. Boer et al., Gluons and the quark sea at high energies: Distributions, polarization, tomography, arXiv: 1108.1713.

[14] J. L. Abelleira Fernandez et al. (LHeC Study Group Collaboration), A large hadron electron collider at CERN: Report on the physics and design concepts for machine and detector, J. Phys. G 39, 075001 (2012).

[15] A. Accardi et al., Electron Ion Collider: The next QCD frontier-Understanding the glue that binds us all, Eur. Phys. J. A 52, 268 (2016).

[16] E. C. Aschenauer et al., The Electron-Ion Collider: Assessing the energy dependence of key measurements, arXiv: 1708.01527.

[17] R. Boussarie, A. V. Grabovsky, L. Szymanowski, and S. Wallon, On the one loop $\gamma^{(*)} \rightarrow q \bar{q}$ impact factor and the exclusive diffractive cross sections for the production of two or three jets, J. High Energy Phys. 11 (2016) 149.

[18] Y. Hagiwara, Y. Hatta, and T. Ueda, Wigner, Husimi, and generalized transverse momentum dependent distributions in the color glass condensate, Phys. Rev. D 94, 094036 (2016).

[19] J. Zhou, Elliptic gluon generalized transverse-momentumdependent distribution inside a large nucleus, Phys. Rev. D 94, 114017 (2016).

[20] X. Ji, F. Yuan, and Y. Zhao, Hunting the Gluon Orbital Angular Momentum at the Electron-Ion Collider, Phys. Rev. Lett. 118, 192004 (2017).

[21] Y. Hatta, Y. Nakagawa, B. Xiao, F. Yuan, and Y. Zhao, Gluon orbital angular momentum at small- $x$, Phys. Rev. D 95, 114032 (2017).

[22] Y. Hagiwara, Y. Hatta, B. W. Xiao, and F. Yuan, Elliptic flow in small systems due to elliptic gluon distributions?, Phys. Lett. B 771, 374 (2017).

[23] S. Bhattacharya, A. Metz, and J. Zhou, Generalized TMDs and the exclusive double Drell-Yan process, Phys. Lett. B 771, 396 (2017).

[24] Y. Hatta, B. W. Xiao, and F. Yuan, Gluon tomography from deeply virtual Compton scattering at small-x, Phys. Rev. D 95, 114026 (2017).
[25] Y. Hagiwara, Y. Hatta, R. Pasechnik, M. Tasevsky, and O. Teryaev, Accessing the gluon Wigner distribution in ultraperipheral $p A$ collisions, Phys. Rev. D 96, 034009 (2017).

[26] J. More, A. Mukherjee, and S. Nair, Wigner distributions for gluons, Eur. Phys. J. C 78, 389 (2018).

[27] A. Rajan, M. Engelhardt, and S. Liuti, Lorentz invariance and QCD equation of motion relations for generalized parton distributions and the dynamical origin of proton orbital angular momentum, arXiv:1709.05770 [Phys. Rev. D (to be published)].

[28] Y. Hagiwara, Y. Hatta, B. W. Xiao, and F. Yuan, Classical and quantum entropy of parton distributions, Phys. Rev. D 97, 094029 (2018).

[29] Y. Hatta, Notes on the orbital angular momentum of quarks in the nucleon, Phys. Lett. B 708, 186 (2012).

[30] Y. Zhao, K. F. Liu, and Y. B. Yang, Orbital angular momentum and generalized transverse momentum distribution, Phys. Rev. D 93, 054006 (2016).

[31] S. Bhattacharya, A. Metz, V. K. Ojha, J. Y. Tsai, and J. Zhou, Exclusive double quarkonium production and generalized TMDs of gluons, arXiv:1802.10550.

[32] D. Boer, T. Van Daal, P. J. Mulders, and E. Petreska, Directed flow from C-odd gluon correlations at small $x$, J. High Energy Phys. 07 (2018) 140.

[33] J. W. Qiu, P. Sun, B. W. Xiao, and F. Yuan, Universal suppression of heavy quarkonium production in pA collisions at low transverse momentum, Phys. Rev. D 89, 034007 (2014).

[34] Z. B. Kang, Y. Q. Ma, and R. Venugopalan, Quarkonium production in high energy proton-nucleus collisions: CGC meets NRQCD, J. High Energy Phys. 01 (2014) 056.

[35] L. D. McLerran and R. Venugopalan, Green's functions in the color field of a large nucleus, Phys. Rev. D 50, 2225 (1994).

[36] I. Balitsky, Operator expansion for high-energy scattering, Nucl. Phys. B463, 99 (1996).

[37] R. Boussarie, A. V. Grabovsky, L. Szymanowski, and S. Wallon, Impact factor for high-energy two and three hets diffractive production, J. High Energy Phys. 09 (2014) 026.

[38] M. Martinez, M. Sievert, and D. Wertepny, Toward initial conditions of conserved charges part I: Spatial correlations of quarks and antiquarks, J. High Energy Phys. 07 (2018) 003.

[39] T. Altinoluk, N. Armesto, G. Beuf, A. Kovner, and M. Lublinsky, Quark correlations in the color glass condensate: Pauli blocking and the ridge, Phys. Rev. D 95, 034025 (2017).

[40] G. T. Bodwin, E. Braaten, and G. P. Lepage, Rigorous QCD analysis of inclusive annihilation and production of heavy quarkonium, Phys. Rev. D 51, 1125 (1995); Erratum, Phys. Rev. D 55, 5853(E) (1997).

[41] H. S. Shao, Y. Q. Ma, K. Wang, and K. T. Chao, Polarizations of $\chi_{c 1}$ and $\chi_{c 2}$ in Prompt Production at the LHC, Phys. Rev. Lett. 112, 182003 (2014).

[42] H. Han, Y. Q. Ma, C. Meng, H. S. Shao, and K. T. Chao, $\eta_{c}$ Production at LHC and Indications on the Understanding of $J / \psi$ Production, Phys. Rev. Lett. 114, 092005 (2015).

[43] M. Butenschoen, Z. G. He, and B. A. Kniehl, $\eta_{c}$ Production at the LHC Challenges Nonrelativistic-QCD Factorization, Phys. Rev. Lett. 114, 092004 (2015). 
[44] P. Hagler, R. Kirschner, A. Schafer, L. Szymanowski, and O. V. Teryaev, Towards a Solution of the Charmonium Production Controversy: $k^{-}$Perpendicular Factorization versus Color Octet Mechanism, Phys. Rev. Lett. 86, 1446 (2001).

[45] S. P. Baranov, A. V. Lipatov, and N. P. Zotov, Prompt charmonia production and polarization at LHC in the
NRQCD with kt-factorization. Part II: $\chi_{c}$ mesons, Phys. Rev. D 93, 094012 (2016).

[46] M. G. A. Buffing, M. Diehl, and T. Kasemets, Transverse momentum in double parton scattering: factorisation, evolution and matching, J. High Energy Phys. 01 (2018) 044. 